\title{
Flow across microvessel walls through the endothelial surface glycocalyx and the interendothelial cleft
}

\author{
M. SUGIHARA-SEKI, T. AKINAGA AND T. ITANO \\ Department of Physics, Kansai University, 3-3-35 Yamate-cho, Suita, Osaka 564-8680, Japan
}

(Received 8 March 2007 and in revised form 9 January 2008)

A mathematical model is presented for steady fluid flow across microvessel walls through a serial pathway consisting of the endothelial surface glycocalyx and the intercellular cleft between adjacent endothelial cells, with junction strands and their discontinuous gaps. The three-dimensional flow through the pathway from the vessel lumen to the tissue space has been computed numerically based on a Brinkman equation with appropriate values of the Darcy permeability. The predicted values of the hydraulic conductivity $L_{p}$, defined as the ratio of the flow rate per unit surface area of the vessel wall to the pressure drop across it, are close to experimental measurements for rat mesentery microvessels. If the values of the Darcy permeability for the surface glycocalyx are determined based on the regular arrangements of fibres with $6 \mathrm{~nm}$ radius and $8 \mathrm{~nm}$ spacing proposed recently from the detailed structural measurements, then the present study suggests that the surface glycocalyx could be much less resistant to flow compared to previous estimates by the one-dimensional flow analyses, and the intercellular cleft could be a major determinant of the hydraulic conductivity of the microvessel wall.

\section{Introduction}

The luminal surface of vascular endothelial cells is covered by a layer of macromolecules referred to as the glycocalyx. Its role as a molecular filter and diffusive barrier has been pointed out to be crucial for microvessel walls to maintain low permeability to macromolecules. In our previous paper (Sugihara-Seki 2006), we developed a theoretical model for transport of solutes and fluid across the endothelial surface glycocalyx, and successfully described the size selective properties of the glycocalyx for solute transport, such as diffusive permeability and reflection coefficient. For the fluid transport, on the other hand, it was indicated that the hydraulic conductivity $L_{p}$, which is defined as the ratio of the flow rate per unit surface area of the vessel wall relative to the pressure drop across it, cannot be accounted for by the glycocalyx property alone. In this paper, we develop a three-dimensional model to describe the hydraulic conductivity $L_{p}$ across the entire endothelial barrier including the endothelial surface glycocalyx and the interendothelial cleft with its tight junction strand. This model is based on the latest detailed ultrastructural studies of venular microvessels in rat mesentery (see figure 1) (Adamson et al. 2004).

In electron micrographs of microvessels in transverse section, the clefts are seen as narrow spaces, typically $14-21 \mathrm{~nm}$ wide, between the outer leaflets of adjoining endothelial cells. The route from the luminal entrance to the abluminal exit along the cleft is interrupted by one or more tight junctions. The tight junctions form lines 




Tissue space

FIGURE 1. Sketch of an interendothelial cleft and surface glycocalyx of a microvessel wall.

of contact between the adjacent cells which can be traced in serial sections to run approximately parallel to the luminal and abluminal surfaces of the endothelium. They appear to act as barriers for water and solute to flow within the cleft. Where the tight junction continuity ends, the distance between cells is observed to open to equal the width of the wide part of the cleft. Water and solutes are expected to bypass the tight junction through these discontinuities or gaps. The spacing between membranes of facing cells is of remarkable uniformity except for tight junctions. Although there is some experimental evidence of the presence of regularly spaced cleft-spanning structures in rat myocardial capillaries (Schulze \& Firth 1992), little information is currently available as to whether such structures fill the entire cleft.

Existing models of the filtration flow in the cleft and through the breaks in the tight junction strand have all been based on the two-dimensional Hele-Shaw flow theory first proposed in Tsay, Weinbaum \& Pfeffer (1989). This approach was suggested by the nearly uniform gap height of the cleft. At the time, there was conflicting evidence as to the nature of the pores in the tight junction strand, whether they were long discontinuities or small slit-like breaks. The latter was suggested by the ultra thin sections of rat heart capillaries (Bundgaard 1984). The paper by Adamson \& Michel (1993) in which the tight junctions of frog mesentery microvessels were serially reconstructed provided convincing evidence that the dominant pathway for water through the cleft was large infrequent, widely spaced breaks (see figure $13 a$ of Tsay et al. 1989). Since Hele-Shaw flow satisfies a potential flow equation, this observation allowed Parker et al. in the Appendix to Adamson \& Michel (1993) to apply conformal mapping techniques to develop an analytical solution for a tight junction strand of zero thickness that was arbitrarily positioned in the depth direction of the cleft. The convergence and divergence of the flow on each side of the tight junction strand could be treated by introducing a correction factor $C$ that described the reduction in flow resistance relative to a one-dimensional flow through the orifice-like break owing to the spreading of streamlines.

In the one-dimensional flow of the plane Poiseuille flow between two parallel plates with separation distance $2 h$, the flow rate per unit length in the span-wise direction is given by $2 h^{3} \Delta p / 3 \mu l_{C}$, where $l_{C}$ is the distance from the vessel lumen to the interstitium, $\Delta p$ is a pressure drop across them and $\mu$ is the fluid viscosity. Thus, if we denote the length of intercellular cleft per unit area of vessel wall as $A$, and the length of the discontinuities expressed as a fraction of the total length of the cleft as $f$, then the hydraulic conductivity for the one-dimensional flow and that for the 
two-dimensional flow can be described as (Adamson \& Michel 1993)

$$
L_{p}^{(1)}=A f \frac{2 h^{3}}{3 \mu l_{C}}, \quad L_{p}^{(2)}=C L_{p}^{(1)},
$$

where superscripts (1) and (2) denote the one-dimensional flow and the twodimensional Hele-Shaw flow, respectively. In the Appendix to Adamson \& Michel (1993), the correction factor $C$ was plotted as a function of the junction position relative to the cleft depth for various lengths of the discontinuities (see figure 10).

This same approach could also be used if there was a fibre matrix in the cleft, provided the pressure at the entrance and exit was uniform, since Darcy flow also satisfies a potential flow equation. A more sophisticated analysis of the Stokes flow around possible cross-bridging fibres in the cleft was developed in Tsay \& Weinbaum (1991) who also showed that the flow through the fibres could be accurately modelled by a Brinkman equation (see equation (2)). This model was extended to treat the diffusion of solutes through a cross-bridging fibre array in Weinbaum, Tsay \& Curry (1992).

Although electron-microscopic studies as early as Luft (1966) had indicated that the surface of vascular endothelium was covered by a glycocalyx, it was not realized until Adamson \& Clough (1992) that this coating was of nearly uniform thickness and completely covering the cleft entrance. To treat this complication, $\mathrm{Fu}$ et al. (1994) proposed a junction-orifice-fibre entrance layer model to describe both the hydraulic and diffusive permeabilities of the entire endothelial layer. They converted the thickness of the surface glycocalyx to an approximate equivalent thickness of matrix inside the cleft by comparing the Darcy permeability of the unbounded fibre matrix with the effective values of the bounded fibre array. It was predicted that the matrix layer of ordered periodic structure of about $100 \mathrm{~nm}$ thickness could provide the hydraulic conductivity $L_{p}$ compatible with experimental observations, and a thicker layer would offer too much hydraulic resistance for frog mesentery capillaries.

Subsequent papers by Weinbaum and coworkers were motivated by the recognition that the classical Starling hypothesis (1896) required a major revision to account for the measured Starling force balance properly (Michel 1997; Weinbaum 1998). These investigators proposed that the Starling forces, which had been widely believed to act globally between plasma and tissue, actually acted just across the endothelial surface glycocalyx. This being the case, the model in Fu et al. (1994) was modified in $\mathrm{Hu} \&$ Weinbaum (1999) to couple the filtration flow to the convective and diffusive transport of albumin through the glycocalyx layer and the underlying cleft with its tight junction. The major feature of this new model was the treatment of the back diffusion of solute from the tissue and the theoretical prediction of the oncotic forces that were measured in the experiments in frog mesentery (Hu et al. 2000) and rat mesentery (Adamson et al. 2004). To accommodate the nonlinear coupling of the water and solute fluxes, these more recent papers attempted to simplify the junction-orifice-fibre matrix model in Fu et al. (1994) by treating the flow through the glycocalyx as a slowly varying one-dimensional flow while retaining the Hele-Shaw description of the flow in the cleft. This model was then further simplified in Zhang et al. (2006) into an equivalent one-dimensional model for the entire layer including the back diffusion from the tissue.

The objectives of the present study are to examine critically some of the important simplifications in the hydrodynamic assumptions used in these previous models. In particular, (i) we examine the slowly varying one-dimensional approximation for the flow through the glycocalyx which neglects the convergence of streamlines at the cleft 
entrance, (ii) we examine the use of a Hele-Shaw approximation for the flow in the cleft which does not satisfy the no-slip boundary conditions on the tight junction strand and the edges of the orifice breaks, and (iii) we also present the first results for a glycocalyx with an inner sieving layer and a more diffuse outer layer as proposed in Pries, Secomb \& Gaehtgens (2000). In addition, we calculate the fluid shear stress acting on the membranes bounding the cleft and forces acting on the tight junction strands, and present detailed solutions for the velocity field at the entrance of the cleft and in the vicinity of the orifice breaks.

We shall present our formulation and methods of analyses in $\S 2$, and the numerical results will be provided in $\S 3$ for the flow structure, the hydraulic conductivity, the fluid shear stresses on the membrane and the forces exerted on the tight junction strand. By comparing the present results with previous model studies and experimental studies, we shall discuss the contribution of the glycocalyx to $L_{p}$ and the corrections necessary for the previous estimates in $\S 4$.

\section{Formulation and methods}

We consider the flow of an incompressible Newtonian fluid across a microvessel wall from the vessel lumen to the tissue space. The pathway of the fluid consists of the endothelial surface glycocalyx and clefts between neighbouring endothelial cells. Figure 1 shows a schematic view of ultrastructures of the interendothelial cleft and the surface glycocalyx (Squire et al. 2001; Weinbaum et al. 2003; Adamson et al. 2004). Adamson et al. (2004) showed from their detailed structural morphology measurements for rat mesentery venules that the clefts between adjacent endothelial cells have uniform width of $2 h=18 \mathrm{~nm}$ on average and there are tight junction strands with discontinuous leakages, or gaps, of mean width $2 d=315 \mathrm{~nm}$ and mean spacing $2 D=3590 \mathrm{~nm}$. It was reported that, on average, the total cleft depth is $l_{C}=411 \mathrm{~nm}$, and the tight junction strand is located at $15 \%$ of the cleft depth or $l_{J}=67 \mathrm{~nm}$ from the luminal cleft opening (table 1). They made simultaneous measurements of the hydraulic conductivity for rat mesentery venules that are on average $L_{p}=1.0-$ $1.3 \times 10^{-7} \mathrm{~cm}\left(\mathrm{cmH}_{2} \mathrm{O}\right)^{-1} \mathrm{~s}^{-1}$. Note that $1 \mathrm{~cm}\left(\mathrm{cmH}_{2} \mathrm{O}\right)^{-1} \mathrm{~s}^{-1} \approx 10^{-4} \mathrm{~m} \mathrm{~Pa}^{-1} \mathrm{~s}^{-1}$.

Based on figure 1 , we have adopted an idealized geometry depicted in figure 2 as a model for the fluid flow pathway across microvessel walls. Endothelial cells are assumed to be rectangular hexahedrons, and the two sidewalls of neighbouring cells facing each other compose the cleft with uniform width $2 h$ and depth $l_{C}$ from the luminal cleft opening to the abluminal cleft exit. The tight junction strand is represented by an impermeable barrier to flow with thickness $\delta$, that lies parallel to the luminal front with depth $l_{J}$ from the luminal cleft entrance, and it has discontinuous gaps with length $2 d$, periodically placed at a spacing of $2 D$ along the tight junction strand. The surface glycocalyx is assumed to cover the entire surface of the endothelial cells including the entrance region to the cleft, and have a constant thickness $l_{G}$. We assume that there is a uniform space of the vessel lumen upstream of the glycocalyx, with thickness $l_{L}$, and there is a uniform tissue space downstream from the cleft exit, with thickness $l_{T}$.

The Cartesian coordinate system $(x, y, z)$ is introduced such that the $(x, y)$-plane is the mid-plane of the cleft, the $(y, z)$-plane corresponds to the luminal surface of the endothelial cells, and the $x$-axis is along the line from the vessel lumen to the tissue space, passing the centre of the gap of the junction strand. Figure 2 shows a periodic unit of the model geometry, which repeats in the $y$ - and $z$-directions. The period $2 D$ in the $y$-direction represents the separation distance between neighbouring gaps 


$\begin{array}{ll}\text { Gap length }^{1} & 2 d=315 \mathrm{~nm} \\ \text { Gap spacing }^{1} & 2 D=3590 \mathrm{~nm} \\ \text { Cleft spacing } & 2 L=10000 \mathrm{~nm} \\ \text { Cleft width }^{1} & 2 h=18 \mathrm{~nm} \\ \text { Cleft length per area vessel wall }^{1} & A=0.10 \mu \mathrm{m}^{-1} \\ \text { Glycocalyx thickness }^{2} & l_{G}=150-400 \mathrm{~nm} \\ \text { Total cleft depth }^{1} & l_{C}=411 \mathrm{~nm} \\ \text { Depth of strand }^{1} & l_{J}=67 \mathrm{~nm} \\ \text { Strand thickness }^{3} & \delta=15 \mathrm{~nm} \\ \text { Vessel lumen thickness } & l_{\mathrm{L}}=150-400 \mathrm{~nm} \\ \text { Tissue space thickness } & l_{\mathrm{T}}=1000 \mathrm{~nm} \\ \text { Endothelial surface glycocalyx } & \\ \quad \text { Fibre radius } & \\ \quad \text { Fibre spacing } & \end{array}$

Darcy permeability for the flow parallel to the core proteins

$$
K_{p}^{G / /}=6.10 \mathrm{~nm}^{2}
$$

Darcy permeability for the flow perpendicular to the core proteins

$$
K_{p}^{G_{\perp}}=3.16 \mathrm{~nm}^{2}
$$

Interendothelial cleft

Fibre radius ${ }^{5}$

Fibre spacing ${ }^{6}$

Darcy permeability

$1.25 \mathrm{~nm}$

$15 \mathrm{~nm}$

$K_{p}^{C}=\infty$ or $32.84 \mathrm{~nm}^{2}$

Tissue space

Darcy permeability $K_{p}^{T}=190 \mathrm{~nm}^{2}$

${ }^{1}$ Adamson et al. (2004), ${ }^{2}$ Weinbaum et al. (2003), ${ }^{3}$ Adamson \& Michel (1993), ${ }^{4}$ Squire et al. (2001), ${ }^{5}$ Shapiro et al. (1995), ${ }^{6}$ Schulze \& Firth (1992), ${ }^{7}$ Levick (1987), ${ }^{8}$ Intaglietta \& de Plomb (1973).

TABLE 1. Parameter values.

of the tight junction strand, and $2 L$ represents the distance between neighbouring clefts. Adamson et al. (2004) measured the perimeters and luminal surface areas of individual endothelial cells by using confocal microscopy techniques, and calculated the mean cleft length per unit surface area of the vessel wall $A$ as $0.100 \mu \mathrm{m}^{-1}$ for rat mesentery venules. Applying this value to the present model, we have $2 L=10 \mu \mathrm{m}$ since $A=2 D /(2 D \times 2 L)=0.100 \mu \mathrm{m}^{-1}$. The parameter values are summarized in table 1 .

We analyse the motion of water from the vessel lumen to the tissue space. Because of comparable sizes of mean distance between adjacent water molecules of about $0.3 \mathrm{~nm}$ and cleft width of $18 \mathrm{~nm}$, the best analysis would be performed based on their molecular nature. One of the most important differences from a continuum approach would be the possible presence of liquid slippage along the interface of the liquid and solid phases (Priezjev \& Troian 2006). Despite a long history of work on this topic as well as recent developments of molecular-dynamics simulations and experiments, there remains a significant debate concerning the validity of slip conditions as well as the degree of slip possible at liquid/solid interfaces. Little is known about the motion of water molecules near biological membranes. Thus, the current study is performed based on continuum mechanics under the conventional no-slip condition on the membrane surface. Future information about the molecular dynamics will help us to improve the present analysis.

We consider the flow of a Newtonian fluid with viscosity $\mu$ from the vessel lumen to the tissue space, when a certain pressure drop $\Delta p$ is prescribed between them. 


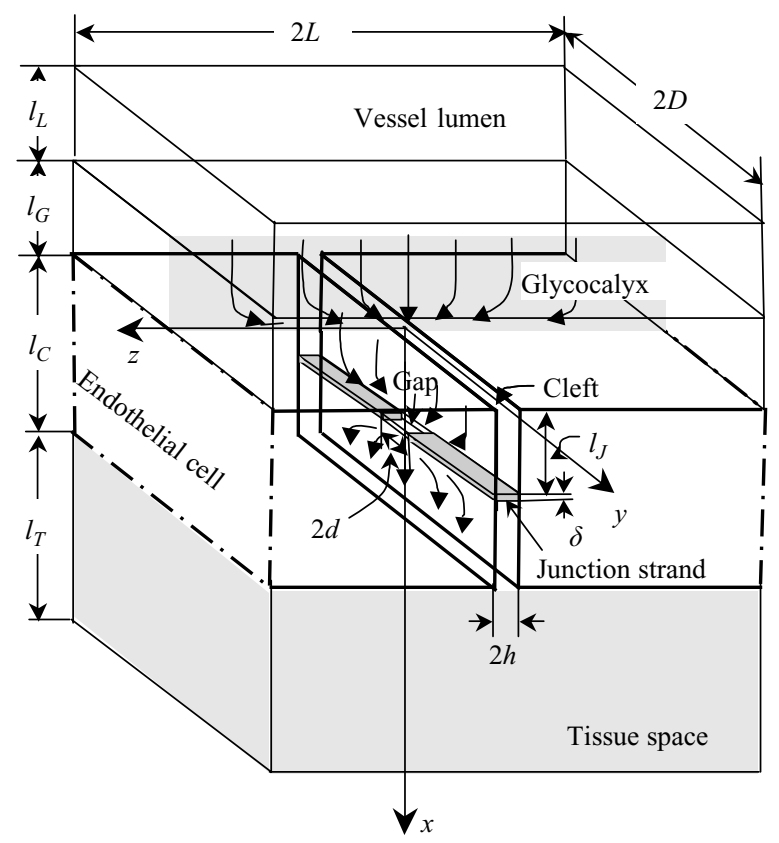

FIGURE 2. Model for a serial pathway of water flow from the vessel lumen to the tissue space through the endothelial surface glycocalyx and the interendothelial cleft. The geometry is repeated in the $y$ - and $z$-directions.

The domain of the numerical computation contains four regions. The first region is the vessel lumen at $-\left(l_{G}+l_{L}\right) \leqslant x \leqslant-l_{G}$. The pressure at $x=-\left(l_{G}+l_{L}\right)$ is given as $p=0$. The second region is the endothelial surface glycocalyx at $-l_{G} \leqslant x \leqslant 0$, the third region is the interendothelial cleft at $0 \leqslant x \leqslant l_{C}$, and the fourth region is the tissue space at $l_{C} \leqslant x \leqslant l_{C}+l_{T}$. The pressure at $x=l_{C}+l_{T}$ is prescribed as $p=-\Delta p$. From the flow rate $J_{v}$ per unit cross-sectional area and the pressure drop $\Delta p$, we calculated the hydraulic conductivity $L_{p}=J_{v} / \Delta p$. Some typical in vivo permeability experiments are performed in the range of $\Delta p$ from about $10 \mathrm{cmH}_{2} \mathrm{O}$ to 50 or $60 \mathrm{cmH}_{2} \mathrm{O}$ (Adamson et al. 2004; Truskey, Yuan \& Katz 2004).

Since the Reynolds number is extremely small, the inertia of the fluid motion can be neglected. The effect of the presence of the fibre matrices in the endothelial surface glycocalyx, the interendothelial cleft and the tissue space was considered approximately by adding a Darcy force term proportional to the fluid velocity to the Stokes equation, called the Brinkman equation (Brinkman 1947):

$$
-\nabla p+\mu \Delta \boldsymbol{u}-\frac{\mu}{K_{p}} \boldsymbol{u}=0
$$

where $\boldsymbol{u}=(u, v, w)$ is the fluid velocity, $p$ is the pressure and $K_{p}$ is the Darcy permeability. This equation has been widely used to represent the viscous flow through fibrous media, from the similarity to the flow through porous media (Damiano et al. 1996; Feng, Ganatos \& Weinbaum 1998; Feng \& Weinbaum 2000; Sugihara-Seki 2004, 2006; Han et al. 2006). We assume the viscosity of water $\mu=0.69 \times 10^{-3} \mathrm{~Pa} \mathrm{~s}^{-1}$ at $37^{\circ} \mathrm{C}$.

The value of $K_{p}$ depends on the fibre geometry. For instance, if we assume the fibres to be circular cylinders with radius $r_{f}$, the Darcy permeability $K_{p}$ for a flow 
perpendicular to a two-dimensional hexagonal array of the circular cylinders can be expressed by using the solutions in Sangani \& Acrivos (1982) as

$$
\frac{K_{p}}{r_{f}^{2}}=\frac{\ln \left(c^{-1 / 2}\right)-0.745+c-c^{2} / 4+O\left(c^{4}\right)}{4 c},
$$

where $c$ is the volume fraction of the cylinders. In terms of spacing $\Delta$ between neighbouring cylinder surfaces instead of $c$, Tsay \& Weinbaum (1991) obtained a useful approximate expression for equation (3):

$$
\frac{K_{p}}{r_{f}^{2}}=0.0572\left(\frac{\Delta}{r_{f}}\right)^{2.377} .
$$

The glycocalyx has a very delicate fragile structure with high water content (Pries et al. 2000; van den Berg, Vink \& Spaan 2003; van den Berg et al. 2006). It is so tenuous that knowledge of its ultrastructure and constitutive behaviour in vivo are very limited. As a result, its value of the Darcy permeability is currently unknown. In the present study, we have estimated the Darcy permeability for the surface glycocalyx $K_{p}^{G}$ based on the detailed structural measurements of the glycocalyx (Squire et al. 2001). From the observation by the autocorrelation imaging techniques, they proposed a structural model where the surface glycocalyx is composed of bushlike clusters of core proteins projecting normally to the surface of the luminal wall (figure 1). Within each cluster, the scattering centres along the core proteins are 10$12 \mathrm{~nm}$ in diameter and their interval is roughly $20 \mathrm{~nm}$ in the directions both parallel and perpendicular to the endothelial surface. If we simplify this model by assuming core proteins to be circular cylinders with radius $r_{f}=6 \mathrm{~nm}$ which align regularly perpendicular to the endothelial luminal surface in a hexagonal array with spacing $\Delta=$ $8 \mathrm{~nm}$, then (3) gives $K_{p}^{G_{\perp}}=3.16 \mathrm{~nm}^{2}$ for the volume fraction $c=2 \pi r_{f}^{2} / \sqrt{3}\left(2 r_{f}+\Delta\right)^{2}=$ 0.326 . Here the superscript $\perp$ indicates the value for the flow perpendicular to the cylinder axis.

For the flow parallel to the cylinder axis, Sugihara-Seki (2006) solved the Stokes equation and estimated the Darcy permeability to be $K_{p}^{G_{/ /}}=6.10 \mathrm{~nm}^{2}$. Corresponding to (3), an approximate analytical representation of the Darcy permeability for parallel flow in the case of low volume fraction $c$ can be deduced from equation (18) in Sparrow \& Loeffler (1959) after some manipulation:

$$
\frac{K_{p}}{r_{f}^{2}}=\frac{2 c-\ln c-c^{2} / 2-1.5}{4 c} .
$$

If we put $c=0.326$, equation (5) gives $K_{p}^{G_{/ /}}=6.07 \mathrm{~nm}^{2}$. This is close to the above estimate in spite of a fairly large value of $c$.

It may be interesting to compare these values of the Darcy permeability with the value obtained from the well-known Carman-Kozeny equation (Happel \& Brenner 1973). In terms of the volume fraction of fibres $c$, the fibre radius $r_{f}$ and the so-called Kozeny constant $k$, this may be written as (Curry \& Michel 1980)

$$
\frac{K_{p}}{r_{f}^{2}}=\frac{(1-c)^{3}}{4 k c^{2}}
$$

Substituting $c=0.326, r_{f}=6 \mathrm{~nm}$ and $k=5.6$ for flow through random orientation of cylinders, equation (6) gives $K_{p}=4.63 \mathrm{~nm}^{2}$, which lies between $K_{p}^{G_{\perp}}=3.16 \mathrm{~nm}^{2}$ and $K_{p}^{G_{/ /}}=6.10 \mathrm{~nm}^{2}$. 
Squire et al. (2001) pointed out that the thickness of the glycocalyx having the ultrastructure of core proteins is no more than $200 \mathrm{~nm}$. On the other hand, the work of Duling and coworkers (Vink \& Duling 1996; Henry \& Duling 1999, 2000) and Damiano and coworkers (Smith et al. 2003; Damiano, Long \& Smith 2004; Long et al. 2004) provide evidence for a $400-500 \mathrm{~nm}$ thick surface layer in capillaries and venules in vivo. Thus, it may be plausible to assume the possible presence of another layer extending further into the vessel lumen than the core proteins. Such a layer, hereinafter called the apical layer, may have constituents different from those of the core-protein layer, and may be composed of adsorbed plasma components and glycosaminoglycans (GAGs) (Pries et al. 2000). Since the permeability property of the apical layer is unknown, we assume, in the present study, constant values of the Darcy permeability for the whole endothelial surface layer including the coreprotein layer and the apical layer, for simplicity, and call the whole surface layer the endothelial surface glycocalyx. We examine the two cases of $l_{G}=150 \mathrm{~nm}$ and $400 \mathrm{~nm}$ as representative values of the glycocalyx thickness. The effect of different values of the Darcy permeability between the two layers on $L_{p}$ will be discussed in $\S 4$.

If there are no cleft-spanning molecules in the intercellular cleft, the Darcy permeability for the cleft would be $K_{p}^{C}=\infty$. In this case, (2) reduces to the Stokes equation. If we assume that the cleft-spanning molecules are placed perpendicular to the cleft surface in a hexagonal array at $15 \mathrm{~nm}$ intervals and their diameter is taken as $2.5 \mathrm{~nm}$ (Schulze \& Firth 1992; Shapiro et al. 1995; Boggon et al. 2002; Adamson et al. 2004), then we have $K_{p}^{C}=32.84 \mathrm{~nm}^{2}$ from (4). It is not certain whether the cleft-spanning molecules fill the entire cleft or not, but it is more likely that the link proteins, VE cadherins, may occur only in localized regions (Thi et al. 2004; Zhang et al. 2006). Thus, in the present study, the case of $K_{p}^{C}=\infty$ is mainly treated and the results of this case are reported in $\S 3$ unless otherwise noted. Some cases of $K_{p}^{C}=32.84 \mathrm{~nm}^{2}$ are also examined to compare with previous studies. For the Darcy permeability in the tissue space, we adopted $K_{p}^{T}=190 \mathrm{~nm}^{2}$ from measurements on mesentery (Intaglietta \& de Plomb 1973; Levick 1987).

In a brief summary of the present analysis, we have solved the Brinkman equation (equation (2)) simultaneously in the whole domain, with the Darcy permeability appropriate to each region such as

$$
K_{p}= \begin{cases}\infty & \text { for }-\left(l_{G}+l_{L}\right)<x<-l_{G}(\text { vessel lumen }) \\ K_{p}^{G_{/ /}}, K_{p}^{G_{\perp}} & \text { for } \left.-l_{G}<x<0 \text { (glycocalyx }\right) \\ K_{p}^{C} & \text { for } 0<x<l_{C}(\text { cleft }) \\ K_{p}^{T} & \text { for } l_{C}<x<l_{C}+l_{T}(\text { tissue space })\end{cases}
$$

where the values of $K_{p}^{G_{/ /}}, K_{p}^{G_{\perp}}, K_{p}^{C}$ and $K_{p}^{T}$ are given in table 1. Boundary conditions are no-slip condition on the surfaces of endothelial cells and tight junction strands. Periodic boundary conditions are adopted in the $y$ - and $z$-directions, and up- and down-stream boundary conditions are

$$
\begin{aligned}
& \partial u / \partial x=0, \quad v=w=0 \quad \text { at } \quad x=-\left(l_{G}+l_{L}\right), l_{C}+l_{T} \\
& p=0 \quad \text { at } \quad x=-\left(l_{G}+l_{L}\right), p=-\Delta p \quad \text { at } \quad x=l_{C}+l_{T}
\end{aligned}
$$

Note that, since (2) is linear in $\boldsymbol{u}$, the flow rate $J_{v}$ is proportional to $\Delta p$ and the hydraulic conductivity $L_{p}=J_{v} / \Delta p$ is independent of $\Delta p$ in the present model. With regard to a choice of the vessel lumen length, $l_{L}$, we found negligible effects on $L_{p}$, which is due to negligible pressure drop across the vessel lumen (see figures 3 and 4). 
(a)

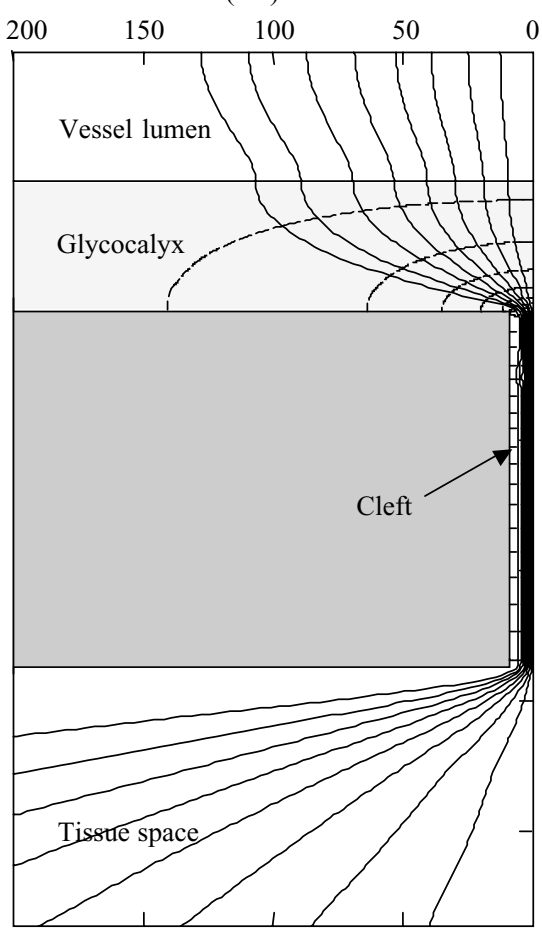

(b)

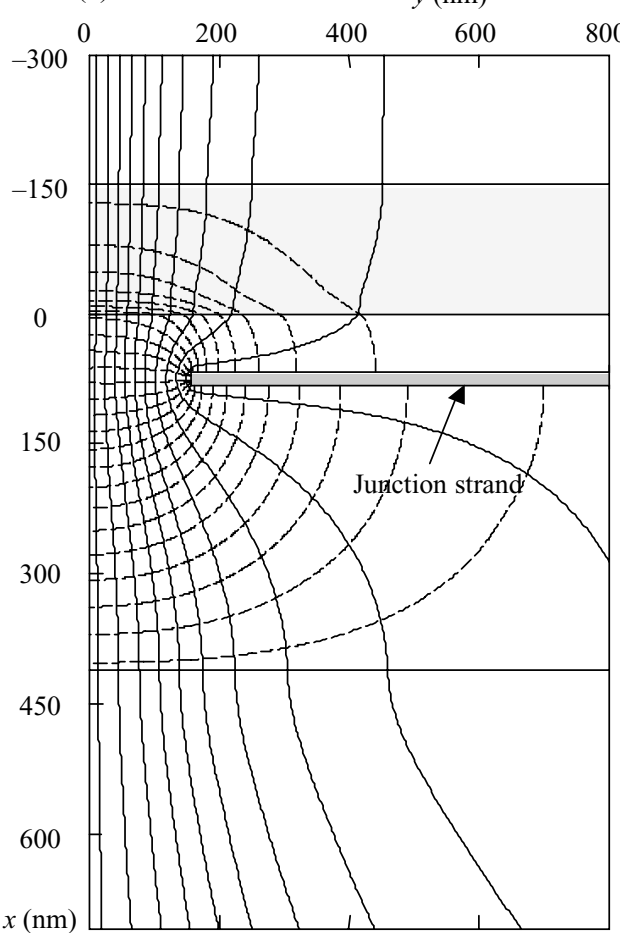

FIGURE 3. Streamlines (solid curves) and pressure contours (dashed curves) in the $(x, z)$-plane (a) and in the $(x, y)$-plane $(b)$, for $l_{G}=150 \mathrm{~nm}, l_{T}=150 \mathrm{~nm}$ and $K_{p}^{C}=\infty$ with the other parameters in table 1 . In $(a)$, the streamlines are drawn in such a way that the flow rates on the $(x, z)$-plane across the mid-cross-section of the gap $\left(x=l_{J}+\delta / 2\right)$ are the same between any two adjacent streamlines. In $(b)$, the streamlines cross the line at $x=l_{J}+\delta / 2$ with an equal interval along the gap of the junction strand.

In our numerical analysis, we have adopted a finite-element spectral method applied to the Brinkman equation and continuity equation. The fluid domain shown in figure 2 was divided into a number of finite elements with hexahedral shapes. Within each element, the velocity and pressure were interpolated by Chebyshev polynomials of $N$ th degree and $(N-1)$ th degree, respectively. Special attention was paid in making elements to the corner regions where singular behaviours are expected in the velocity and the pressure. To overcome this difficulty, we adopted the gradual $h$-refinement method, in which sizes of elements were progressively decreased toward the corners with size ratio $=0.15$ (Babuska \& Suri 1994; Karniadakis \& Sherwin 2005). The finite-element scheme was formulated in terms of the primitive variables based on the variational principle $\delta J=0$ (Sugihara-Seki 1996, 2004). Here, the variational functional was chosen as

$$
J=\int_{\Omega}\left[\mu \boldsymbol{e}^{2}-p(\nabla \cdot \boldsymbol{u})+\frac{\mu}{K_{p}} \frac{\boldsymbol{u}^{2}}{2}\right] \mathrm{d} x \mathrm{~d} y \mathrm{~d} z-\int_{\partial \Omega} \boldsymbol{\tau}_{n} \cdot \boldsymbol{u} \mathrm{d} S,
$$

where $\boldsymbol{e}=\left(\nabla \boldsymbol{u}+(\nabla \boldsymbol{u})^{t}\right) / 2, \Omega$ represents the fluid domain, and $\delta \Omega$ is the boundary where the fluid stress exerted on it is prescribed as $\boldsymbol{\tau}_{n}$. In the present computation, the $x$-components of $\boldsymbol{\tau}_{n}$ were given on the up- and down-stream cross-sections at $x=-\left(l_{G}+l_{L}\right)$ and $l_{C}+l_{T}$, from (8). 

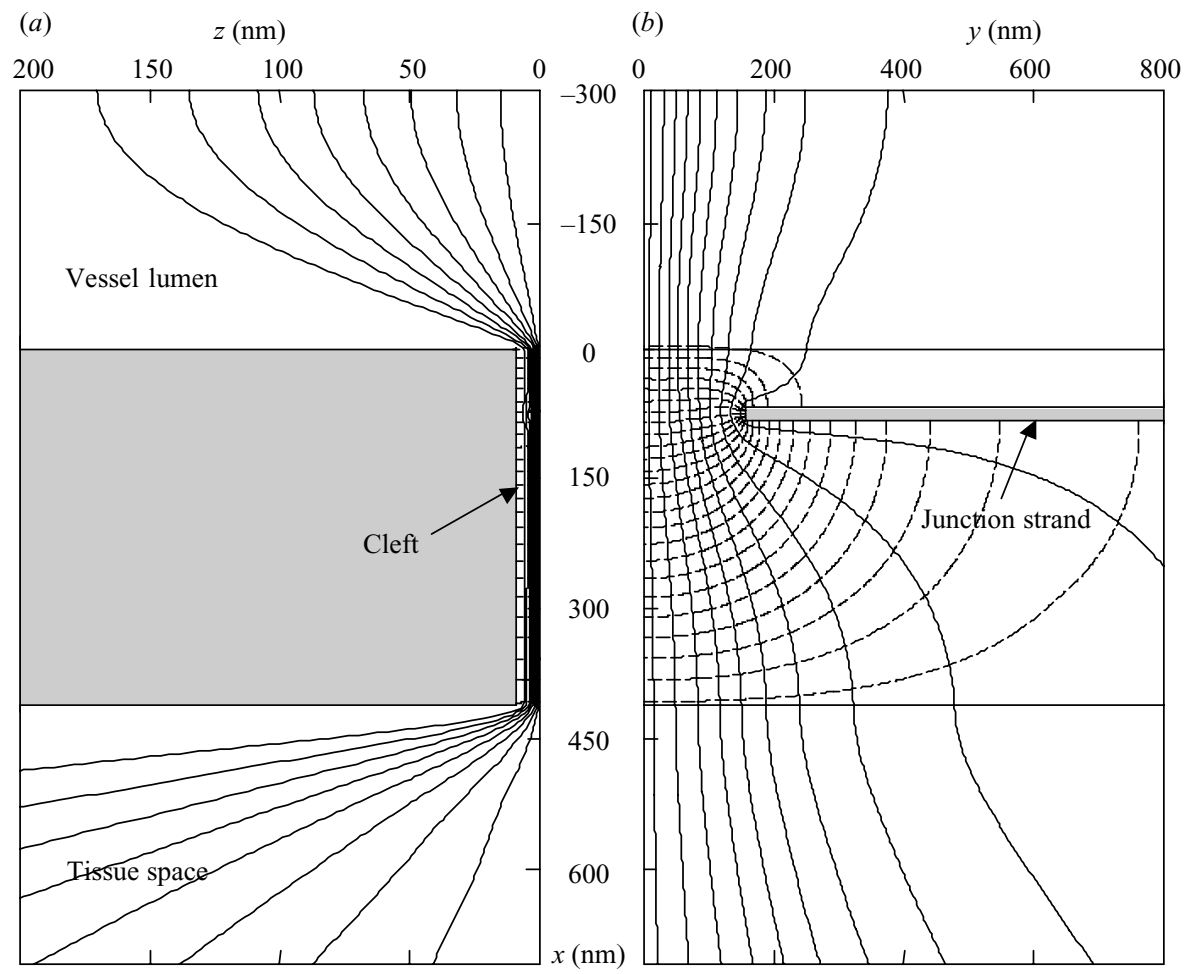

FIGURE 4. Streamlines (solid curves) and pressure contours (dashed curves) in the $(x, z)$-plane $(a)$ and in the $(x, y)$-plane $(b)$, for $l_{G}=0, l_{T}=300 \mathrm{~nm}$ and $K_{p}^{C}=\infty$ with the other parameters in table 1.

In order to ascertain the validity of the computation, we calculated the flow rate $Q$ across several cross-sections along the pathway, and estimated its variation relative to the inlet value, $Q(x) / Q_{0}$, where $Q_{0}=Q\left(-\left(l_{G}+l_{L}\right)\right)$ represents the flow rate across the most upstream cross-section in the vessel lumen at $x=-\left(l_{G}+l_{L}\right)$. For various degrees $N$ of Chebyshev polynomials, we calculated the relative flow rate $Q(x) / Q_{0}$, along the $x$-axis at $x=-l_{G}$ (glycocalyx surface), $x=0$ (luminal cleft entrance), $x=l_{J}$ (entrance of the gap of the tight junction), $x=l_{J}+\delta$ (exit of the gap), $x=l_{C}$ (abluminal cleft exit) and $x=l_{C}+l_{T}$ (tissue space). For the cases of $N=2$, deviations from unity are significant at the luminal cleft entrance and the abluminal exit, but they are progressively decreased as the polynomial degrees are increased. For the cases of $N=4$ and 183 elements, the relative errors are within $0.5 \%$ for all cross-sections, and we have adopted $N=4$ in the present study. In a representative computation, the CPU time for the computation of one configuration with double precision was approximately 6 hours on the FACOM HPC.

\section{Results}

A representative result of our numerical computations is shown in figure 3 , where streamlines and pressure contours are plotted in the $(x, z)$-plane and $(x, y)$-plane, for $l_{G}=150 \mathrm{~nm}$ and $l_{T}=150 \mathrm{~nm}$. In figure $3(b)$ the streamlines in the glycocalyx are almost parallel to the $x$-axis, whereas in figure 3(a) a converging flow from the vessel lumen into the cleft entrance is clearly shown within the glycocalyx. The corresponding 


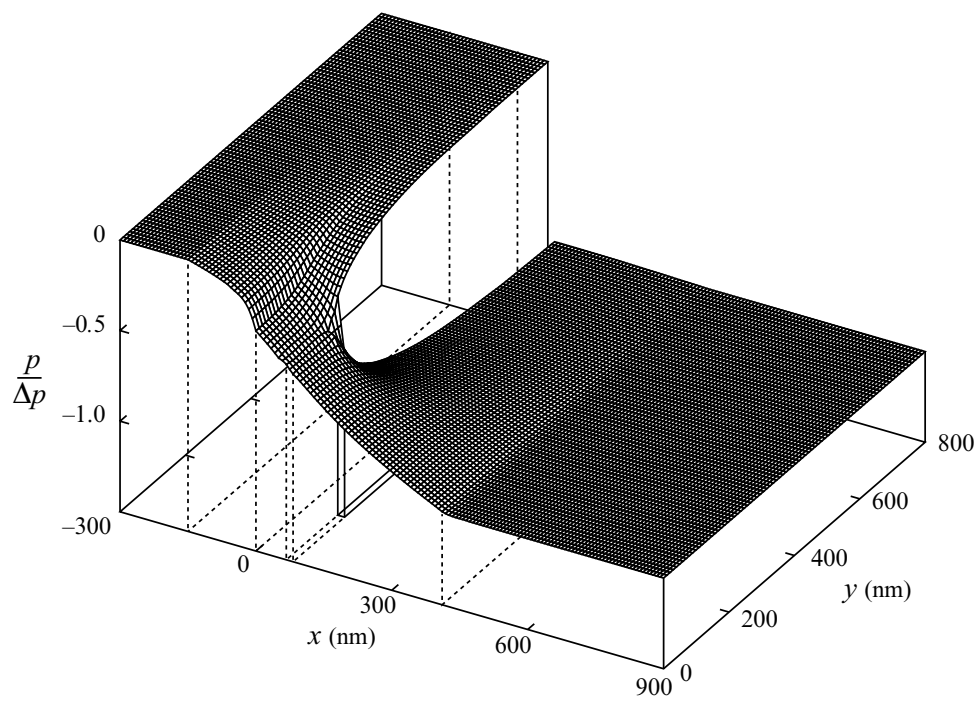

FIGURE 5. Oblique view of the pressure distribution in the $(x, y)$-plane, for $l_{G}=150 \mathrm{~nm}$, $l_{T}=150 \mathrm{~nm}$ and $K_{p}^{C}=\infty$ with the other parameters in table 1.

plots in the absence of the glycocalyx are shown in figure 4 , for reference. We note from figures 3 and 4 that the pressure gradients are present within the glycocalyx both in the $y$ - and $z$-directions as well as in the $x$-direction, which is in marked contrast to the vessel lumen and the tissue space. Figure 4(a) shows that, without the glycocalyx, the streamlines in the vessel lumen extend almost radially from the luminal cleft entrance, similar to those in the tissue space. In comparing the outermost streamlines in figures $3(b)$ and $4(b)$, we notice that the flow from the vessel lumen enters the cleft entrance spreading more widely from the $x$-axis in the presence of the glycocalyx than without the glycocalyx, which will be confirmed below from figure 6 .

The streamlines in the cleft shown in figures $3(b)$ and $4(b)$ demonstrate how the fluid flow passes through the gap of the junction strand in the mid-plane of the cleft. They are similar downstream of the junction strand, while they show some difference upstream of the junction strand, owing to the presence or absence of the glycocalyx. A close look at the cleft region suggests that a nearly potential-type flow may be realized, since the streamlines and pressure contours seem almost perpendicular to each other (see equation (12)).

Figure 5 shows an oblique view of the pressure distribution in the $(x, y)$-plane, corresponding to figure $3(b)$. It is clearly illustrated that the pressure varies mildly in the endothelial glycocalyx except just near the cleft entrance, and most pressure drop occurs in the cleft. The pressure decreases monotonically along the $x$-axis, while, apart from the gap $(y>d)$, the pressure is almost constant in either region upstream or downstream of the tight junction strand. Across the junction strand, there is an abrupt change in pressure, or pressure jump. The considerable pressure jump across the junction strand was previously reported by $\mathrm{Hu} \&$ Weinbaum (1999).

Along the cleft entrance at $x=0$, the pressure has a minimum at $y=0$, and it gradually increases toward an asymptote as $y$ increases from 0 . This behaviour of the pressure variation along the $y$-axis is shown in figure $6(b)$, and the pressure variation along the $z$-axis is shown in figure $6(a)$, for various values of the glycocalyx 


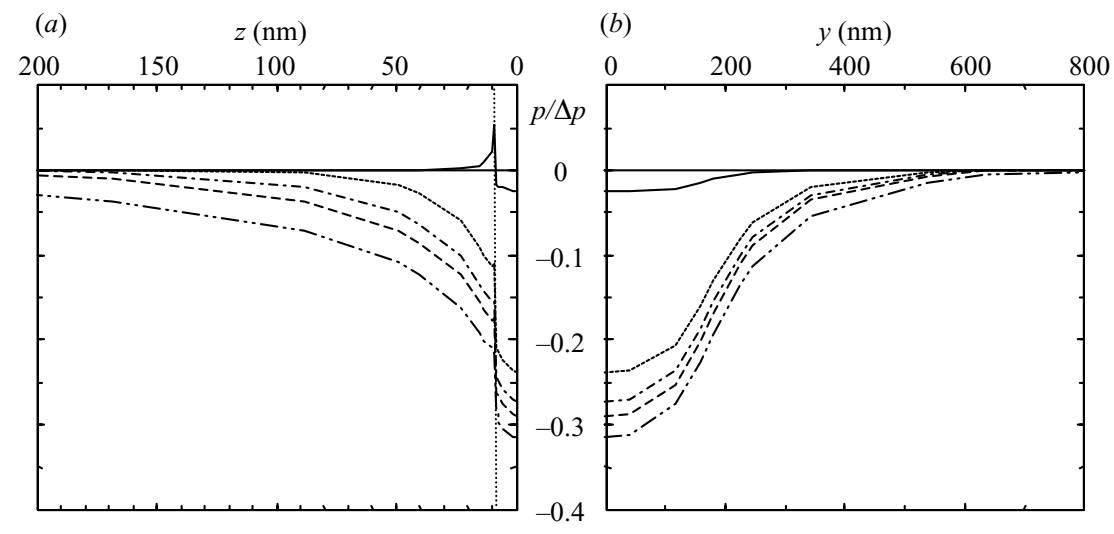

(c)

$(d)$
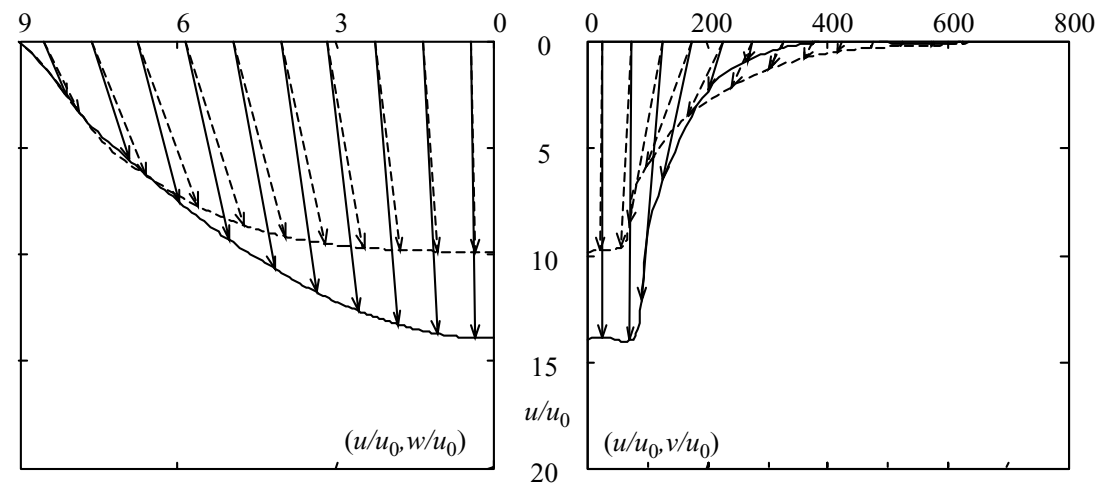

FIgURE 6. The pressure profiles at the cleft entrance along the $z$-axis $(a)$ and the $y$-axis $(b)$, at $K_{p}^{C}=\infty$ for various glycocalyx thicknesses with the other parameters in table 1 . The solid curves represent the case of $l_{G}=0$, the dotted curves $l_{G}=50 \mathrm{~nm}$, the dash-dotted curves $l_{G}=100 \mathrm{~nm}$, the dashed curves $l_{G}=150 \mathrm{~nm}$, and the dash-double dotted curves $l_{G}=400 \mathrm{~nm}$. The velocity vectors at the cleft entrance along the $z$-axis $(c)$ and the $y$-axis $(d)$, at $K_{p}^{C}=\infty$ in the absence of the glycocalyx (solid arrows) and in the presence of the glycocalyx with $l_{G}=150 \mathrm{~nm}$ (dashed arrows), with the other parameters in table 1 . In $(c)$ and $(d)$, the velocity is normalized by the mean velocity in the cleft, $u_{0}=J_{v} L / h$.

thickness. Our computations showed that the depression of the pressure curve near the origin (the centre of the cleft entrance) becomes shallower and narrower as the glycocalyx becomes thinner. Figure $6(b)$ reveals that, in the limit of $l_{G}=0$, i.e. with no glycocalyx, the deviation of the pressure from the asymptotic value is small and it is negligible except in a narrow region corresponding to the opening length of the gap of the junction strand, i.e. $y \leqslant d$. In figure 6(a), a sharp change in pressure is seen at $z=h(=9 \mathrm{~nm})$, corresponding to the corner of the cleft entrance. In particular, the abrupt increase in pressure there was previously observed for Stokes flow through a periodic infinite-slit array in an unbounded plane wall (Hasimoto 1958).

The velocity vectors $(u, w)$ and $(u, v)$ at the cleft entrance normalized by the mean velocity in the cleft, $u_{0}=J_{v} L / h$, are plotted along the $z$-axis in figures $6(c)$ and along the $y$-axis in figure $6(d)$. The solid arrows represent the velocity vectors in the absence of the glycocalyx, and the dashed arrows in the presence of the glycocalyx with $l_{G}=150 \mathrm{~nm}$. Owing to the normalization of the velocity, larger velocity near the 


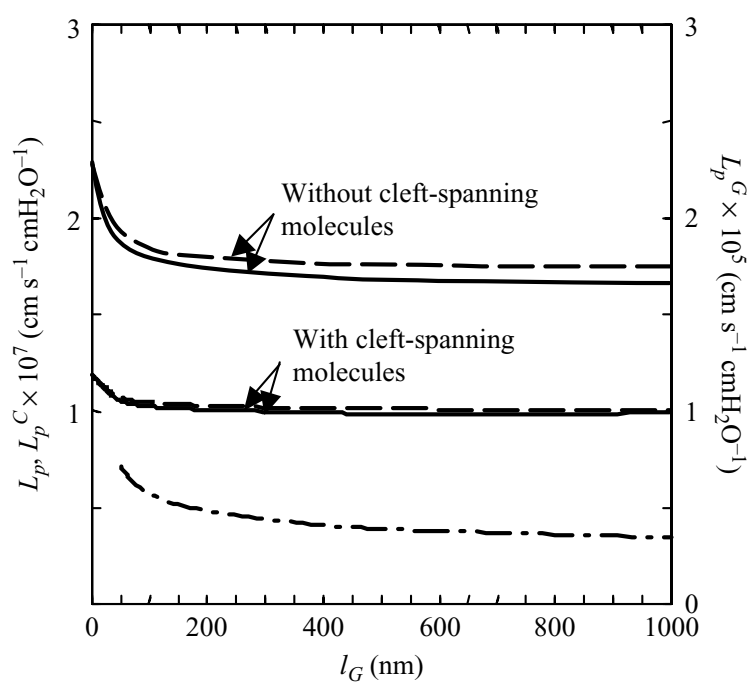

FiguRE 7. Effect of the glycocalyx thickness on the hydraulic conductivity $L_{p}, L_{p}^{C}$ and $L_{p}^{G}$, with and without cleft-spanning molecules. The solid curves represent $L_{p}$, dashed curves $L_{p}^{C}$ and dash-dotted curves $L_{p}^{G}$.

centreline ( $x$-axis) with no glycocalyx than that with the glycocalyx indicates that, without the glycocalyx, the fluid flow into the cleft entrance is more concentrated near the $x$-axis, compared to the case with the glycocalyx, as already noted from figures $3(b)$ and $4(b)$. This variation of the velocity profile at the cleft entrance affects the flow structure in the cleft, which results in variations of the hydraulic conductivity with the glycocalyx thickness, as shown below.

With the use of the velocity data obtained and the pressure drop prescribed, the hydraulic conductivity $L_{p}=J_{v} / \Delta p$ was calculated, for various parameter values. As seen in figure 5, variations in pressure are very small within the vessel lumen $\left(-\left(l_{G}+l_{L}\right) \leqslant x \leqslant-l_{G}\right)$ and within the tissue space $\left(l_{C} \leqslant x \leqslant l_{C}+l_{T}\right)$, so that $\Delta p$ is nearly equal to the pressure difference across the surface glycocalyx and the cleft, i.e. $\Delta p \approx \bar{p}\left(-l_{G}\right)-\bar{p}\left(l_{C}\right)$. Here, $\bar{p}\left(x_{0}\right)$ represents the mean pressure averaged over the cross-section at $x=x_{0}$. Figure 7 shows our predicted values of $L_{p}$ as a function of the glycocalyx thickness $l_{G}$, with and without cleft-spanning molecules. The other parameters are shown in table 1 . For $K_{p}^{C}=\infty$, the present computations lead to $L_{p}=1.75 \times 10^{-7} \mathrm{~cm}\left(\mathrm{cmH}_{2} \mathrm{O}\right)^{-1} \mathrm{~s}^{-1}$ for $l_{G}=150 \mathrm{~nm}$, and $L_{p}=1.69 \times 10^{-7} \mathrm{~cm}\left(\mathrm{cmH}_{2} \mathrm{O}\right)^{-1} \mathrm{~s}^{-1}$ for $l_{G}=400 \mathrm{~nm}$. In the presence of the cleftspanning molecules $\left(K_{p}^{C}=32.84 \mathrm{~nm}^{2}\right)$, we had $L_{p}=1.01 \times 10^{-7} \mathrm{~cm}\left(\mathrm{cmH}_{2} \mathrm{O}\right)^{-1} \mathrm{~s}^{-1}$ for $l_{G}=150 \mathrm{~nm}$, and $L_{p}=0.99 \times 10^{-7} \mathrm{~cm}\left(\mathrm{cmH}_{2} \mathrm{O}\right)^{-1} \mathrm{~s}^{-1}$ for $l_{G}=400 \mathrm{~nm}$. These values are within the range of the experimental measurements and close to the mean value $1.0-1.3 \times 10^{-7} \mathrm{~cm}\left(\mathrm{cmH}_{2} \mathrm{O}\right)^{-1} \mathrm{~s}^{-1}$ for rat mesentery venules (Adamson et al. 2004).

The hydraulic conductivity for the glycocalyx alone is calculated by the ratio of the flow rate and the pressure difference across the glycocalyx, i.e. between its luminal surface and the entrance to the cleft just behind it, such as $L_{p}^{G}=J_{v} /\left(\bar{p}\left(-l_{G}\right)-\bar{p}(0)\right)$. In this case, $\bar{p}(0)$ represents the mean pressure averaged over the luminal cleft entrance, i.e. $-D \leqslant y \leqslant D$ and $-h \leqslant z \leqslant h$ at $x=0$. Similarly, the hydraulic conductivity for the cleft is given by $L_{p}^{C}=J_{v} /\left(\bar{p}(0)-\bar{p}\left(l_{C}\right)\right)$. Since we consider a serial pathway of the 


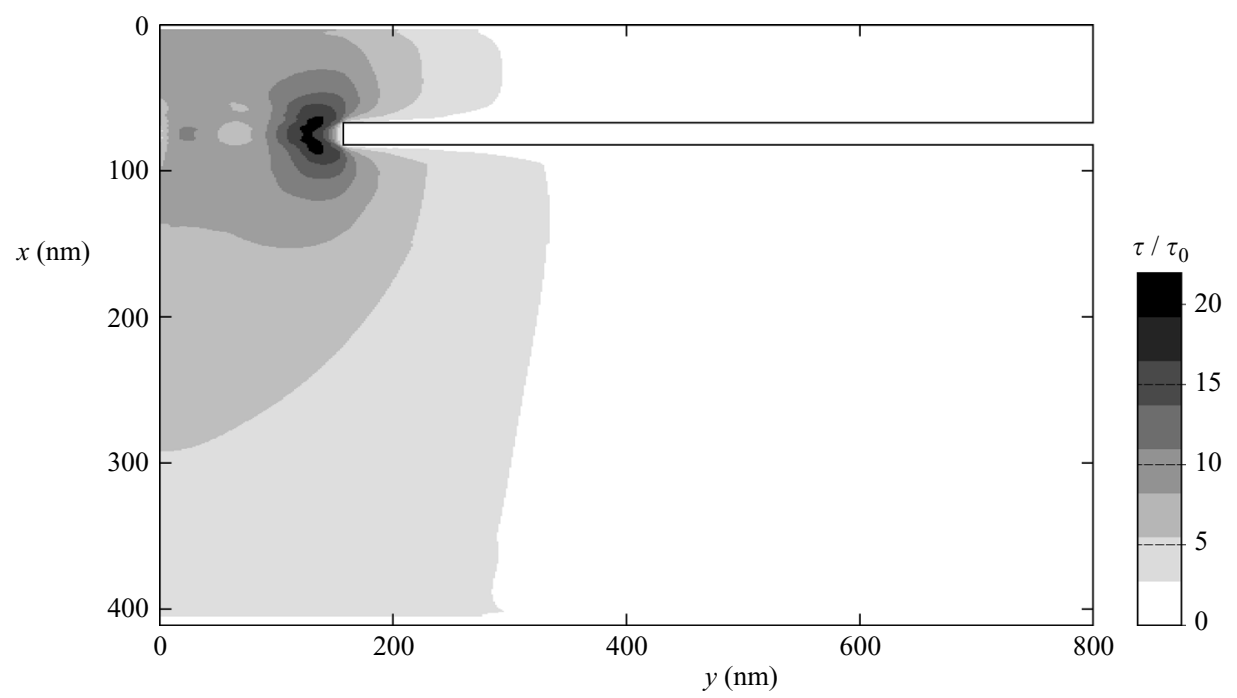

FIgURE 8 . The distributions of shear stresses exerted by the fluid flow on the surface of the endothelial cell in the cleft at $z= \pm h$, for $l_{G}=150 \mathrm{~nm}$ and $K_{p}^{C}=\infty$ with the other parameters in table 1 . The magnitudes of the shear stresses $\tau$ are normalized by $\tau_{0}=3 \mu u_{0} / h$, where $u_{0}$ represents the mean velocity in the cleft $u_{0}=J_{v} L / h$.

glycocalyx and the cleft for the fluid flow, we have the relationship:

$$
\frac{1}{L_{p}}=\frac{1}{L_{p}^{G}}+\frac{1}{L_{p}^{C}}
$$

where $\left(L_{p}\right)^{-1},\left(L_{p}^{G}\right)^{-1}$ and $\left(L_{p}^{C}\right)^{-1}$ represent the resistance to flow in the vessel wall, the glycocalyx and the cleft, respectively. In figure 7, the variations of $L_{p}^{C}$ and $L_{p}^{G}$ with the glycocalyx thickness $l_{G}$ are also plotted. Since the values of $L_{p}^{G}$ for the glycocalyx are two orders of magnitude larger than the corresponding values of $L_{p}^{C}$, it is shown that the values of $L_{p}$ are mainly determined by $L_{p}^{C}$. Our computation predicts that the contributions of the glycocalyx to the total flow resistance are only $3.4 \%$ and $4.1 \%$ for $l_{G}=150 \mathrm{~nm}$ and $400 \mathrm{~nm}$, respectively, if the cleft-spanning molecules are absent $\left(K_{p}^{C}=\infty\right)$. The corresponding values are even smaller if the cleft-spanning molecules are present $\left(K_{p}^{C}=32.84 \mathrm{~nm}^{2}\right)$. Comparing the values of the $L_{p}$ curve for $100 \mathrm{~nm}<l_{G}<500 \mathrm{~nm}$ with its $y$-intercept suggests that the removal of the glycocalyx may increase $L_{p}$ by about $30 \%$ for $K_{p}^{C}=\infty$, and about $15 \%$ for $K_{p}^{C}=32.84 \mathrm{~nm}^{2}$. This increase in $L_{p}$ following the removal of the glycocalyx is mostly due to the variation of the flow structure in the cleft, rather than the loss of the flow resistance in the glycocalyx. Figure 7 shows that the values of $L_{p}$ depend only weakly on the glycocalyx thickness for $l_{G}>100 \mathrm{~nm}$, which results from a minor contribution of the glycocalyx to the flow resistance.

The fluid stresses exerted on the endothelial surface were calculated by using the velocity data. Figure 8 shows a representative example of the shear stress distribution on the surface of the intercellular cleft $\left(\right.$ at $z= \pm h$ ) for $l_{G}=150 \mathrm{~nm}$. Here, the magnitudes of the wall shear stress $\tau=\left.\mu \sqrt{(\partial u / \partial z)^{2}+(\partial v / \partial z)^{2}}\right|_{z= \pm h}$ are normalized by the wall shear stress exerted by the plane Poiseuille flow between two parallel plates with separation distance $2 h$, i.e. $\tau_{0}=3 \mu u_{0} / h$. For a representative flow rate $J_{v}=10^{-8} \mathrm{~m} \mathrm{~s}^{-1}$, we have $u_{0}=5.6 \times 10^{-6} \mathrm{~m} \mathrm{~s}^{-1}$ and $\tau_{0}=1.28 \mathrm{~Pa}$, for $h=9 \mathrm{~nm}$. 
The luminal surface of the endothelial cell is continuously exposed to the shear stress of blood flow which is of the order of 1-2 Pa for postcapillary venules (Lipowsky 1995), that may be actually transmitted via the glycocalyx to the cytoskeleton of the endothelial cell (Smith et al. 2003; Long et al. 2004). Comparing with this value, we see from figure 8 that the wall shear stresses exerted on the cleft surface of the endothelial cell are extremely high. The shear stresses are concentrated in the gap region, especially near its ends $\left(l_{J} \leqslant x \leqslant l_{J}+\delta\right.$ and $\left.|y| \sim d\right)$. This is caused by the velocity profile in the gap region, having a minimum at the gap centre, and a maximum near the gap edge with a sharp decline to 0 at the edge to satisfy the no-slip condition. This characteristic velocity profile was previously reported by Zeng \& Weinbaum (1994), where they analysed a Stokes flow through a parallel-walled channel obstructed by a thin planar barrier with a periodically spaced rectangular orifice. It was reported that for moderate and large ratios of channel height to orifice width $(=h / d$ in the present study), the velocity profile in the orifice has the maximum at its centre, just like the Poiseuille flow, whereas a decrease in the ratio shifts the peak position toward the edge of the orifice. Apparently, the present geometry corresponds to the latter, since $h / d=0.0571 \ll 1$ (see table 1 ).

It is increasingly recognized that the endothelial glycocalyx serves a critical role in the transmission of the fluid shear stress to the actin cytoskeleton and in the initiation of intracellular signalling (Thi et al. 2004; Weinbaum, Tarbell \& Damiano 2007). Although the role of the fluid shear stresses in the cleft is not clear, the concentrated shear stresses shown in figure 8 suggest that they may have some effect on the tight junction itself, such as modulating the opening of the pores for small solutes. With regard to the shear stress on the other surfaces of the endothelial cell, the present study shows that the fluid filtration between the vessel lumen and the tissue space may exert the much smaller shear stresses on the luminal side $(x=0)$ and the abluminal side $\left(x=l_{C}\right)$ than those on the cleft surface $(z= \pm h)$.

It may be interesting to estimate how much of the pressure force applied to the cleft, $\Delta p(2 h)(2 D)$, is taken up by shear stresses on the cleft surface. The integrations of the $x$-component of the stresses over the cleft surface and the junction strand surface show that most pressure force (approximately 90\%) acts on the tight junction strand, and the remainder of the force is taken up by the shear stress exerted on the cleft surface. This may be anticipated from figure 5, where the pressure on the upstream-side of the tight junction strand is nearly equal to the most upstream pressure $(=0)$ and the pressure on its downstream-side is close to the most downstream pressure $(=-\Delta p)$, resulting in the force exerted on the tight junction strand being $\Delta p(2 h)(2 D-2 d)$. Accordingly, the fraction of the pressure force taken up by the pressure difference across the tight junction strand is roughly estimated to be $(2 D-2 d) / 2 D \sim 0.9$, since $d / D=0.0877$ (see Table 1). If we substitute the pressure difference $\Delta p=10 \mathrm{cmH}_{2} \mathrm{O}$ and the cleft width $2 h=18 \mathrm{~nm}$, then the corresponding force on the tight junction strand per unit length along the strand is approximately $\Delta p(2 h)=1.8 \times 10^{-5} \mathrm{~N} \mathrm{~m}^{-1}$.

\section{Discussion}

\subsection{Comparison to previous model studies}

As noted in $\S 1, \mathrm{Fu}$ et al. (1994) combined the endothelial surface glycocalyx and the cleft-spanning molecules in their model, and treated the entire matrix layer as if it existed in the entrance region of the wide part of the cleft. In the entrance matrix layer, they assumed a regular array of fine fibres with radius $r_{f}=0.6 \mathrm{~nm}$ 
and spacing $\Delta=7 \mathrm{~nm}$, corresponding to GAG side-chains. The Darcy permeability is estimated to be $K_{p}=7.08 \mathrm{~nm}^{2}$ from (4). It was shown that the matrix layer of ordered periodic structure with about $100 \mathrm{~nm}$ thickness could be compatible with experimental observations for frog mesentery capillaries, $L_{p}=2.0 \times 10^{-7} \mathrm{~cm}\left(\mathrm{cmH}_{2} \mathrm{O}\right)^{-1} \mathrm{~s}^{-1}$. With the glycocalyx covering the entire endothelial surface, Weinbaum and colleagues used the quasi-one-dimensional flow approximation within the glycocalyx and obtained the measured $L_{p}$ when the glycocalyx thickness is $150 \mathrm{~nm}$ and $r_{f}=0.6 \mathrm{~nm}$, $\Delta=7 \mathrm{~nm}$ (Hu \& Weinbaum 1999; Hu et al. 2000; Weinbaum et al. 2003). Using $r_{f}=6 \mathrm{~nm}, \Delta=8 \mathrm{~nm}$ for core proteins and the same geometry with the current study for the rat mesentery microvessels, Adamson et al. (2004) predicted that $L_{p}$ is equal to $1.24 \times 10^{-7} \mathrm{~cm}\left(\mathrm{cmH}_{2} \mathrm{O}\right)^{-1} \mathrm{~s}^{-1}$ for $l_{G}=150 \mathrm{~nm}$ in the presence of the cleftspanning molecules, and about twice of this value in the absence of the cleft-spanning molecules. Quite recently, Zhang et al. (2006) have indicated that the quasi-onedimensional flow approximation in the glycocalyx with the use of $K_{p}^{G}=9.24 \mathrm{~nm}^{2}$ provides $L_{p}=1.3 \times 10^{-7} \mathrm{~cm}\left(\mathrm{cmH}_{2} \mathrm{O}\right)^{-1} \mathrm{~s}^{-1}$ for $l_{G}=150 \mathrm{~nm}$ for the same structural parameters as the present study, free of any microstructure in the cleft.

The difference of the present study from these previous estimates results mainly from the three-dimensional flow structures in the glycocalyx (see figure 3), in spite of using comparable values of the Darcy permeability for the glycocalyx. The one-dimensional flow approximation is suitable in the $y$-direction, whereas it is inappropriate in the $z$ direction, as shown in figure $3(a)$. The converging flow into the luminal cleft entrance in the $z$-direction may markedly increase the flow rate for a given pressure difference across the glycocalyx. Under the quasi-one-dimensional flow approximation, Zhang et al. (2006) showed that along the $x$-axis, about half of the pressure drop occurs across the glycocalyx and half occurs in the cleft, for $l_{G}=150 \mathrm{~nm}$ and $K_{p}^{G}=9.24 \mathrm{~nm}^{2}$. It was also shown that the pressure drop across the glycocalyx averaged over the entire cleft length is $10 \%$ of the total pressure drop across the endothelial cell. In comparison, the present study predicts for the same parameter values except $K_{p}^{G_{/ /}}=6.10 \mathrm{~nm}^{2}$ and $K_{p \perp}^{G}=3.16 \mathrm{~nm}^{2}$ that about $30 \%$ of the pressure drop occurs across the glycocalyx along the $x$-axis (see dashed curves in figure 6), and the pressure drop across the glycocalyx averaged over the entire cleft length is only $3 \%$ of the total pressure drop. The present study suggests much smaller contribution of the surface glycocalyx to the hydraulic conductivity than the previous estimates, primarily owing to substantial spreading of the flow within the glycocalyx.

\subsection{Effects of the Darcy permeability of the glycocalyx on the hydraulic conductivity}

If one-dimensional flow is assumed in the glycocalyx flowing directly into the cleft entrance with no spreading, the Darcy law provides the pressure drop across the glycocalyx thickness as $\Delta p^{G}=\mu U l_{G} / K_{p}^{G}$, where $U$ is the velocity of the onedimensional flow. If we further assume that the flow is uniform, i.e. $U$ is constant along the length of the cleft entrance, then the velocity $U$ is related to the fluid flux per unit area of the endothelial luminal surface $J_{v}$ as $U=J_{v} /(2 h A)$. Thus, $L_{p}^{G}$ for the uniform one-dimensional flow is represented by $L_{p}^{G}=J_{v} / \Delta p^{G}=K_{p}^{G} 2 h A / \mu l_{G}$. Using this value, we here introduce a correction factor $C^{G}$, corresponding to (1) for the cleft, which represents the hydraulic conductivity for the glycocalyx relative to the value obtained by the one-dimensional flow approximation:

$$
L_{p}^{G(1)}=A K_{p}^{G} \frac{2 h}{\mu l_{G}}, \quad L_{p}^{G(3)}=C^{G} L_{p}^{G(1)} .
$$


(a)

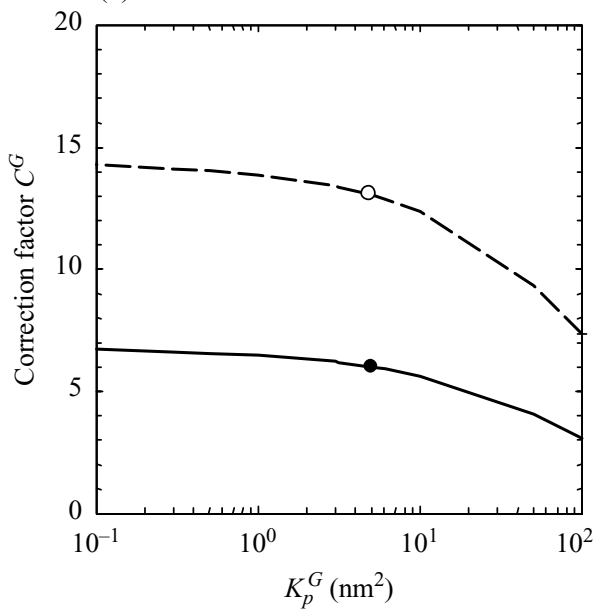

(b)

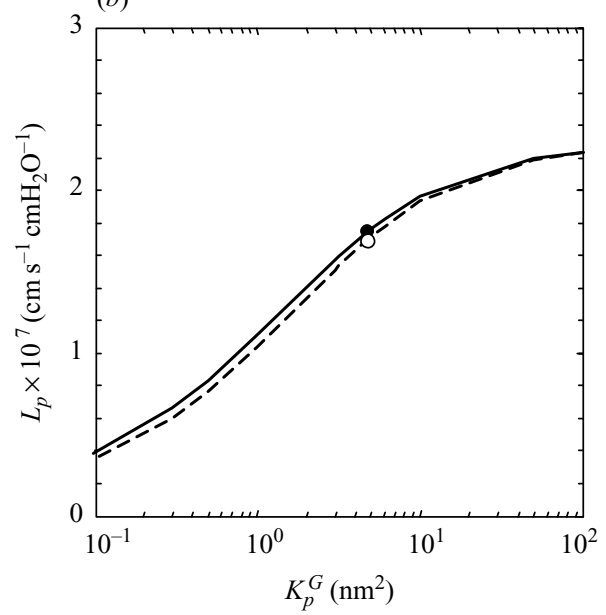

Figure 9. (a) Correction factor $C^{G}$ in (11) as a function of the isotropic Darcy permeability of the glycocalyx $K_{p}^{G}$, at $l_{G}=150 \mathrm{~nm}$ (solid curve) and $400 \mathrm{~nm}$ (dashed curve) for $K_{p}^{C}=\infty$ with the other parameters in table 1. (b) Hydraulic conductivity $L_{p}$ as a function of the isotropic Darcy permeability of the glycocalyx $K_{p}^{G}$, at $l_{G}=150 \mathrm{~nm}$ (solid curve) and $400 \mathrm{~nm}$ (dashed curve) for $K_{p}^{C}=\infty$ with the other parameters in table 1 . In $(a)$ and $(b)$, the filled circles and open circles represent the corresponding values for $K_{p}^{G_{/ /}}=6.10 \mathrm{~nm}^{2}$ and $K_{p}^{G_{\perp}}=3.16 \mathrm{~nm}^{2}$, when isotropic $K_{p}^{G}=4.9 \mathrm{~nm}^{2}$ and $4.8 \mathrm{~nm}^{2}$ are substituted into (11), respectively.

For $l_{G}=150 \mathrm{~nm}$ or $400 \mathrm{~nm}$, we have $L_{p}^{G(1)}=5.5$ or $2.1 \times 10^{-7} \mathrm{~cm}\left(\mathrm{cmH}_{2} \mathrm{O}\right)^{-1} \mathrm{~s}^{-1}$, respectively, when $K_{p}^{G}=3.16 \mathrm{~nm}^{2}$ and $A=10^{-1} \mu \mathrm{m}^{-1}$. For $K_{p}^{G}=6.10 \mathrm{~nm}^{2}$, the corresponding values are $L_{p}^{G(1)}=10.6$ or $4.0 \times 10^{-7} \mathrm{~cm}\left(\mathrm{cmH}_{2} \mathrm{O}\right)^{-1} \mathrm{~s}^{-1}$. Assuming isotropic $K_{p}^{G}\left(=K_{p}^{G_{/ /}}=K_{p}^{G_{\perp}}\right)$, we have used the present model to compute the hydraulic conductivity for the glycocalyx $L_{p}^{G(3)}$ as well as that for the microvessel wall $L_{p}$, and the obtained values of $C^{G}$ and $L_{p}$ are plotted as functions of $K_{p}^{G}$, in figures $9(a)$ and $9(b)$, respectively.

Figure $9(a)$ shows that $C^{G} \approx 6-7$ for $l_{G}=150 \mathrm{~nm}$ in a rather wide range of $K_{p}^{G}$ from $0.1 \mathrm{~nm}^{2}$ to almost $10 \mathrm{~nm}^{2}$, and $C^{G} \approx 13-14$ in the same range of $K_{p}^{G}$ for $l_{G}=400 \mathrm{~nm}$. For an anisotropic case such as $K_{p}^{G_{/ /}}=6.10 \mathrm{~nm}^{2}$ and $K_{p}^{G_{\perp}}=3.16 \mathrm{~nm}^{2}$, the correction factors $C^{G}$ for $l_{G}=150 \mathrm{~nm}$ and $400 \mathrm{~nm}$ are found to lie on the corresponding solid and dashed curves, respectively, if we adopt an isotropic value of $K_{p}^{G}=4.9$ and $4.8 \mathrm{~nm}^{2}$ in the evaluation of $L_{p}^{G(1)}$ by (11).

Figure $9(b)$ shows our predicted values of $L_{p}$ for the vessel wall with the variation of isotropic $K_{p}^{G}$, at $l_{G}=150 \mathrm{~nm}$ and $400 \mathrm{~nm}$. We note that the solid and dashed curves are comparable, indicating that the values of $L_{p}$ are almost independent of the glycocalyx thickness, as already seen in figure 7 . When the value of $K_{p}^{G}$ is increased from $0.1 \mathrm{~nm}^{2}$ to $100 \mathrm{~nm}^{2}$ by three orders of magnitude, figure $9(b)$ indicates that the subsequent

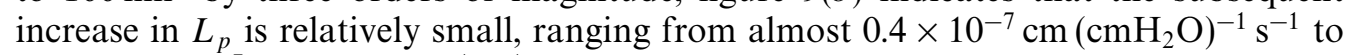
about $2 \times 10^{-7} \mathrm{~cm}\left(\mathrm{cmH}_{2} \mathrm{O}\right)^{-1} \mathrm{~s}^{-1}$.

No direct measurements have been made of the Darcy permeability for the endothelial surface glycocalyx. The values in table $1, K_{p}^{G_{/ /}}=6.10 \mathrm{~nm}^{2}$ and $K_{p}^{G_{\perp}}=3.16 \mathrm{~nm}^{2}$, were determined based on the detailed structural measurements which proposed regular arrangements of core proteins with $r_{f}=6 \mathrm{~nm}$ and $\Delta=8 \mathrm{~nm}$ (Squire et al. 2001; 
Weinbaum et al. 2003). For the core protein geometry adopted in the present study, Sugihara-Seki (2006) made a fluid-mechanical analysis on the motion of a solute between regularly arranged core proteins and estimated the reflection coefficient. The predicted values of the reflection coefficient for various sized solutes are close to experimental measurements, accounting satisfactorily for the observed molecular filtering. For example, the reflection coefficient to solutes corresponding to serum albumin with Stokes radius $=3.6 \mathrm{~nm}$ is predicted to be as high as 0.8 . This estimate explains the experimental measurements much better than previous analyses. Zhang et al. (2006) also reached a similar conclusion using the same geometry of the core proteins. Experimental studies have shown that, despite large variations of $L_{p}$ depending on types of microvessels, various microvessels with fenestrated or continuous endothelium exhibit similar values of the reflection coefficient. This similarity in size selectivity of different types of microvessel walls can be explained well by an idea of the fibre matrix theory that the glycocalyx forms the primary molecular sieve for plasma proteins (Curry \& Michel 1980). Thus, the accordance of the reflection coefficient obtained from the present model with experimental observations may lend support to the present model structures adopted for the glycocalyx.

There may be several factors affecting the value of $K_{p}^{G}$, which was estimated based on the regular arrangement of core proteins. Although we have assumed a rigid structure of the core proteins, resulting in constant values of $K_{p}^{G}$, the flexible property of the glycocalyx as well as the adsorption of plasma proteins may induce a local variation in solid-volume fraction in the glycocalyx, which may alter its permeability property. In addition, surface charges on the molecular filaments of the glycocalyx (Vink \& Duling 2000; Damiano \& Stace 2002) and a possible presence of interconnecting molecules between core proteins would also affect $K_{p}^{G}$.

Among these factors, we here briefly consider the effect of the possible presence of the apical layer at the luminal surface of the core-protein layer on the hydraulic conductivity. As a preliminary study to examine this effect, we modify the present model to include the apical layer, in such a way that the space of the vessel lumen $\left(-\left(l_{G}+l_{L}\right)<x<-l_{G}\right)$ is replaced by the apical layer with a Darcy permeability $K_{p}^{A}$, allowing its variation independent of $K_{p}^{G}$. For the apical layer thickness $l_{L}=300 \mathrm{~nm}$ and $l_{G}=150 \mathrm{~nm}$, i.e. total thickness of the endothelial surface layer being $450 \mathrm{~nm}$, our computation showed that a decrease in $K_{p}^{A}$ from $10 \mathrm{~nm}^{2}$ to $0.1 \mathrm{~nm}^{2}$ reduces $L_{p}$ from


and $K_{p}^{G_{\perp}}=3.16 \mathrm{~nm}^{2}$. These values are not much different from our obtained value of $L_{p}=1.75 \times 10^{-7} \mathrm{~cm}\left(\mathrm{cmH}_{2} \mathrm{O}\right)^{-1} \mathrm{~s}^{-1}$ in the presence of the core-protein layer alone without the apical layer. Thus, it is suggested that the effect of the apical layer on the hydraulic conductivity could be minor when the Darcy permeability of the apical layer is comparable or larger than that of the core-protein layer. Even when $K_{p}^{A}=1 \mathrm{~nm}^{2}$, the contribution of the endothelial surface layer with thickness $450 \mathrm{~nm}$ to the whole resistance is predicted to be about $6 \%$.

\subsection{Effects of the glycocalyx removal on the hydraulic conductivity}

Adamson (1990) demonstrated that enzymatic removal of the surface glycocalyx, using pronase, increased the hydraulic conductivity of frog mesentery capillaries from $2.0 \times 10^{-7} \mathrm{~cm}\left(\mathrm{cmH}_{2} \mathrm{O}\right)^{-1} \mathrm{~s}^{-1}$ to $4.9 \times 10^{-7} \mathrm{~cm}\left(\mathrm{cmH}_{2} \mathrm{O}\right)^{-1} \mathrm{~s}^{-1}$ by 2.5 -fold. This is the only experiment which tried to isolate the contribution of the surface glycocalyx from the whole vessel wall. It is not clear to what extent pronase perfusion degraded the glycocalyx, or affected structures of the cleft and the tight junction strand. However, if we postulate here that the glycocalyx was completely removed with no effect on the 
cleft and tight junction structures, then this in vivo experiment implies that there is a 2.5 times difference between the values of $L_{p}$ with and without the glycocalyx. In the present analysis, in contrast, a comparison of the $L_{p}$ values at $l_{G}=0$ and $l_{G}>150 \mathrm{~nm}$ in figure 7 suggests that the removal of the glycocalyx would enhance $L_{p}$ by about $30 \%$ at $K_{p}^{C}=\infty$. The reason of this discrepancy is not apparent. One cause may be the values of the Darcy permeability for the glycocalyx adopted.

Here, we examine the possibility of adopting a value of $K_{p}^{G}$ which would be pertinent to the in vivo experiment, focusing on the $y$-intercept of the $L_{p}$ curve in figure 7, i.e. $L_{p}=2.28 \times 10^{-7} \mathrm{~cm}\left(\mathrm{cmH}_{2} \mathrm{O}\right)^{-1} \mathrm{~s}^{-1}$ at $l_{G}=0$. To achieve a 2.5 times smaller value of this, i.e. $L_{p}=0.9 \times 10^{-7} \mathrm{~cm}\left(\mathrm{cmH}_{2} \mathrm{O}\right)^{-1} \mathrm{~s}^{-1}$ in the presence of the glycocalyx, we know from figure 9 (b) that $K_{p}^{G} \approx 0.6 \mathrm{~nm}^{2}$. In other words, if we adopted $K_{p}^{G}=0.6 \mathrm{~nm}^{2}$ instead of $K_{p}^{G / /}=6.10 \mathrm{~nm}^{2}$ and $K_{p}^{G_{\perp}}=3.16 \mathrm{~nm}^{2}$, our analysis suggests that the removal of the glycocalyx would increase $L_{p}$ by 2.5 times. However, this value of $K_{p}^{G}$ is far too small to account for experimental measurements of the reflection coefficient to solutes. Actually, for $K_{p}^{G}=0.6 \mathrm{~nm}^{2}$, equation (3) provides the spacing between adjacent fibre surfaces as $\Delta=3.9 \mathrm{~nm}$ and $2.7 \mathrm{~nm}$ for the fibre radius $r_{f}=6 \mathrm{~nm}$ and $0.6 \mathrm{~nm}$, respectively. Similarly, (5) yields $\Delta=3.1 \mathrm{~nm}$ and $2.0 \mathrm{~nm}$ for $r_{f}=6 \mathrm{~nm}$ and $0.6 \mathrm{~nm}$, respectively. These values are even smaller than a half of $\Delta=8 \mathrm{~nm}$ observed by Squire et al. (2001) and adopted in the present study. Apparently, they are too small for serum albumin (radius $=3.6 \mathrm{~nm}$ ) to pass through the spacing. The resulting values of the reflection coefficient would be close to unity for the solute with radius larger than at least $2 \mathrm{~nm}$, which seems inconsistent with measurements of the reflection coefficient (Michel \& Curry 1999).

Unpublished studies (J. Tarbell, personal communication), in which heparinase III instead of pronase has been used to remove the surface glycocalyx in cultured bovine aortic endothelial cell (BAEC) monolayers show only a $38 \%$ increase in the hydraulic conductivity and a $260 \%$ increase for pronase digestion similar to the in vivo study by Adamson (1990). Heparinase is known to degrade selectively heparin sulfate of the glycocalyx (Pahakis et al. 2007). An application of pronase with higher concentration $\left(0.125 \mathrm{mg} \mathrm{ml}^{-1}\right)$ greatly enhanced the hydraulic conductivity of BAEC monolayers. These results suggest some effects of this non-specific enzyme on the tight junction structure, although there is a possibility that the cell-surface chemistry in cultured cells in vitro may be different from the glycocalyx in vivo. Future detailed experimental studies using various enzymes are expected to clarify the contribution of the surface glycocalyx to the hydraulic conductivity of microvessel walls in vivo.

\subsection{The correction factory for the hydraulic conductivity of the clef}

For the flow in the cleft, all previous model studies starting with Tsay et al. (1989) including Adamson et al. (2004) adopted a two-dimensional approximation, where the flow is equivalent to the classical Hele-Shaw flow of a Newtonian fluid in the absence of the cleft-spanning molecules. In the two-dimensional flow analyses, the velocity $(u, v)$ in the cleft is expressed as $u(x, y, z)=u *(x, y)\left(1-z^{2} / h^{2}\right)$ and $v(x, y, z)=v^{*}(x, y)\left(1-z^{2} / h^{2}\right)$, which satisfy the no-slip condition at $z= \pm h$. The velocity in the cleft mid-plane, $\left(u^{*}, v^{*}\right)$, is linearly related to the local gradient in the averaged pressure $p *(x, y)$ as

$$
\left(u^{*}, v^{*}\right)=-K(\partial / \partial x, \partial / \partial y) p^{*},
$$


where $K$ is a constant such that

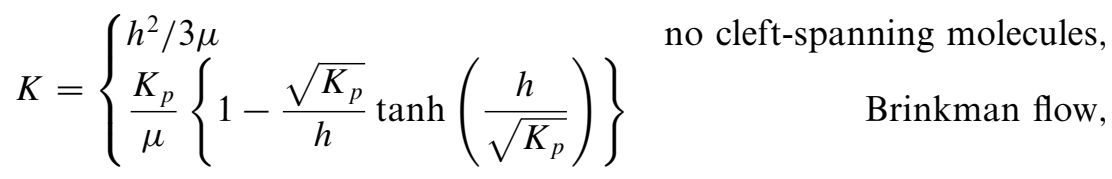

(Phillips, Parker \& Wang 1994). A combination of (12) with the continuity equation leads to the two-dimensional Laplace equation for the pressure:

$$
\nabla^{2} p^{*}=0
$$

Phillips et al. (1994) solved (14) analytically in the domain of $0 \leqslant x \leqslant l_{C}$ subject to the boundary conditions of the uniform constant pressures at the cleft entrance and exit, together with the no-penetrating condition on the surface of the tight junction:

$p^{*}=1 \quad$ on $x=0, p^{*}=0 \quad$ on $x=l_{C}, \partial p^{*} / \partial x=0 \quad$ on $x=\left(l_{J}+\delta / 2\right)_{ \pm},|y| \geqslant d$.

In this analysis, the thickness of the tight junction is neglected and ()$_{ \pm}$represents the luminal and abluminal sides of the tight junction strand.

The solutions of this analysis were summarized in terms of the correction factor $C$, which represents the flow rate of the two-dimensional flow relative to a onedimensional flow through a rectangular cross-section of the gap of the tight junction, for a given pressure drop (Adamson \& Michel 1993). By substituting $f=d / D$ in (1), we have for the flow of a Newtonian fluid in the absence of the cleft-spanning molecules,

$$
L_{p}^{C(1)}=\frac{2 A h^{3} d}{3 \mu l_{C} D}, \quad L_{p}^{C(2)}=C L_{p}^{C(1)} .
$$

In the Appendix to Adamson \& Michel (1993), Parker et al. plotted $C$ as a function of the cleft geometry. Correspondingly, we have computed $C *=L_{p}^{C} / L_{p}^{C(1)}$, where $L_{p}^{C}$ represents the hydraulic conductivity for the three-dimensional Newtonian flow in the present study, with $K_{p}^{C}=\infty$. In figure 10, we plotted $C$ and $C *$ as a function of the junction position from the luminal cleft entrance relative to the cleft depth, $v=\left(l_{J}+\delta / 2\right) / l_{C}$, for various gap lengths relative to the gap separation, $d / D$. The cleft geometry in table 1 corresponds to $v=0.181$ and $d / D=0.0877$. The $C *$ curves in figure 10 represent the cases of $K_{p}^{G / /}=6.10 \mathrm{~nm}^{2}, K_{p}^{G_{\perp}}=3.16 \mathrm{~nm}^{2}$, and $K_{p}^{T}=190 \mathrm{~nm}^{2}$. This asymmetry of the up- and down-stream values of the Darcy permeability reflects in the asymmetry of the $C *$ curves with respect to the centreline $v=0.5$, while the solid curves of $C$ are symmetric owing to the symmetric boundary condition (15).

Similarly, the correction factor, $C^{* *}=L_{p}^{C} / L_{p}^{C(1)}$, can be defined for the Brinkman flow with the use of the following $L_{p}^{C(1)}$ :

$$
L_{p}^{C(1)}=\frac{2 A h K_{p}\left\{1-\frac{\sqrt{K_{p}}}{h} \tanh \left(\frac{h}{\sqrt{K_{p}}}\right)\right\}}{\mu l_{C}} \frac{d}{D} .
$$

This expression is easily obtained from (13). In figure 10, $C^{* *}$ for the Brinkman flow with $K_{p}^{C}=32.84 \mathrm{~nm}^{2}$ is also plotted by dash-dotted curves, for $K_{p}^{G_{/ /}}=6.10 \mathrm{~nm}^{2}$, $K_{p}^{G_{\perp}}=3.16 \mathrm{~nm}^{2}$ and $K_{p}^{T}=190 \mathrm{~nm}^{2}$. Figure 10 clearly demonstrates that the $L_{p}^{C}$ values for the three-dimensional flow are distinctly different from those for one-dimensional or two-dimensional flows, especially for small values of $d / D$, although the flow in the cleft shown by the streamlines and the pressure contours in figures 3 and 4 


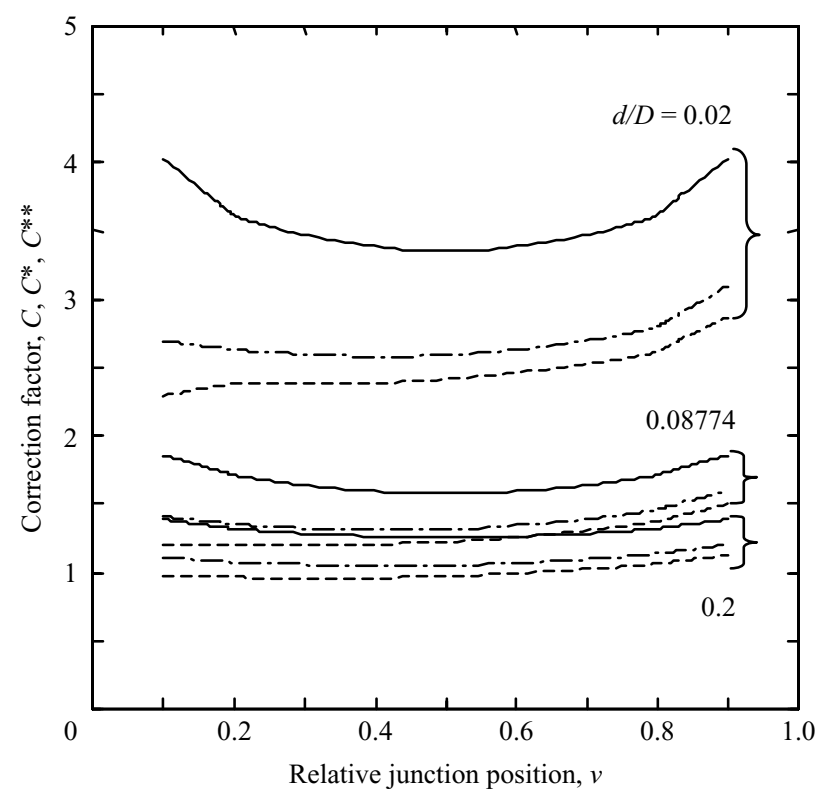

Figure 10. Correction factor $C$ (solid curves), $C *$ (dotted curves) and $C^{* *}$ (dash-dotted curves) $v$ s. relative junction position $v=\left(l_{J}+\delta / 2\right) / l_{C}$, for relative gap length $d / D=0.02,0.08774$ and 0.2 , or $2 d=71.8 \mathrm{~nm}, 315 \mathrm{~nm}$, and $718 \mathrm{~nm}$ at $2 D=3590 \mathrm{~nm}$.

ressembles the two-dimensional potential flow. Generally, $C^{*}$ and $C^{* *}$ are smaller than the corresponding values of $C$, because the three-dimensional flow has a larger resistance to flow owing to the no-slip condition on the surface of the tight junction strand. For $d / D=0.2$, the values of $C^{*}$ and $C^{* *}$ are as low as unity, which may be attributed to non-uniform pressure distributions at the cleft entrance and exit, whose mean pressures, not the local pressures in the region $|y| \leqslant d$, are used to evaluate $L_{p}^{C}$ in the present analysis (see figures 5 and 6 ). For the parameter values shown in table $1, C=1.74, C *=1.21$ and $C^{* *}=1.36$, which are considerably different from each other.

The present method of analysis on the three-dimensional flow will be useful in examining the coupled movement of water and solutes in the cleft, which is closely related to the revised Starling principle proposed by Michel (1997) and Weinbaum (1998). The water flow though the gap of the tight junction strand is expected to affect significantly the back diffusion of solute from the tissue into the luminal side of the cleft, as noted in $\S 1$. This issue is left for a future study.

\section{Concluding remarks}

Using a mathematical model for steady fluid flow through a serial pathway consisting of the endothelial surface glycocalyx and the endothelial intercellular cleft with junction strands and their discontinuous gaps, we analysed three-dimensional flow of the fluid across the microvessel wall and estimated the hydraulic conductivity. If the values of the Darcy permeability for the glycocalyx are determined based on the regular arrangements of fibres with $r_{f}=6 \mathrm{~nm}$ and $\Delta=8 \mathrm{~nm}$, then the present study predicts the hydraulic conductivity close to the experimental measurements for rat mesentery microvessels. A key conclusion may be that the endothelial surface 
glycocalyx could have a much smaller contribution to the flow resistance than does the intercellular cleft. If further information regarding the ultrastructure and composition of the fibre matrices in the glycocalyx as well as the cleft structures becomes available, this idealized model could be improved to provide a more realistic description of the water flow across the microvessel wall.

The authors thank Drs J. Tarbell and S. Weinbaum for providing unpublished experimental data (J. T.) and valuable comments (S. W.). This research was supported in part by Grants-in-Aid for Scientific Research (B) 16360093 and 19360090.

\section{REFERENCES}

Adamson, R. H. 1990 Permeability of frog mesenteric capillaries after partial pronase digestion of the endothelial glycocalyx. J. Physiol. 428, 1-13.

Adamson, R. H. \& Clough, G. 1992 Plasma proteins modify the endothelial cell glycocalyx of frog mesenteric microvessel. J. Physiol. 445, 473-486.

Adamson, R. H. \& Michel, C. C. 1993 Pathways through the intercellular clefts of frog mesenteric capillaries. J. Physiol. 466, 303-327.

Adamson, R. H., Lenz, J. F., Zhang, X., Adamson, G. N., Weinbaum, S. \& Curry, F. E. 2004 Oncotic pressures opposing filtration across non-fenestrated rat microvessles. J. Physiol. 557, 889-907.

BABUSKA, I. \& SURI, M. 1994 The p and h-p versions of the finite element method, basic principles and properties. SIAM Rev. 36, 578-632.

van Den Berg, B. M., Vink, H. \& SpaAn, J. A. E. 2003 The endothelial glycocalyx protects against myocardial edema. Circ. Res. 92, 592-594.

van den Berg, B. M., Nieuwdorp, M., Stroes, E. S. G. \& VinK, H. 2006 Glycocalyx and endothelial (dys) function: from mice to men. Pharmacol. Rep. 58, Suppl. 75-80.

Boggon, T. J., Murray, J., Chappuis-Flament, S., Wong, E., Gumbiner, B. M. \& Shapiro, L. 2002 $\mathrm{C}$-cadherin ectodomain structure and implications for cell adhesion mechanisms. Science 296, $1308-1313$.

Brinkman, H. C. 1947 A calculation of the viscous force exerted by a flowing fluid in a dense swarm of particles. Appl. Sci. Res. A 1, 27-34.

BUNDGAARD, M. 1984 The three-dimensional organization of tight junctions in a capillary endothelium revealed by serial-section electron micrscopy. J. Ultrastruct. Res. 88, 1-17.

Curry, F. E. \& Michel, C. C. 1980 A fibre matrix model of capillary permeability. Microvasc. Res. 20, 96-99.

Damiano, E. R. \& Stace, T. M. 2002 A mechano-electrochemical model of radial deformation of the capillary glycocalyx. Biophys. J. 82, 1153-1175.

Damiano, E. R., Duling, B. R., Ley, K. \& Skalak, T. C. 1996 Axisymmetric pressure-driven flow of rigid pellets through a cylindrical tube lined with a deformable porous wall layer. J. Fluid Mech. 314, 163-189.

Damiano, E. R., Long, D. S. \& SMith, M. L. 2004 Estimation of viscosity profiles using velocimetry data from parallel flows of linearly viscous fluids. J. Fluid Mech. 512, 1-19.

Feng, J. \& Weinbaum, S. 2000 Lubrication theory in highly compressible porous media: the mechanics of skiing, from red cells to humans. J. Fluid Mech. 422, 281-317.

Feng, J., Ganatos, P. \& Weinbaum, S. 1998 Motion of a sphere near planar confining boundaries in a Brinkman medium. J. Fluid Mech. 375, 265-296.

Fu, B. M., Weinbaum, S., Tsay, R. Y. \& Curry, F. E. 1994 A junction-orifice-fibre entrance layer model for capillary permeability: application to frog mesenteric capillaries. Trans. ASME J. Biomech. Engng 116, 502-513.

Han, Y., Weinbaum, S., SpaAn, J. A. E. \& VinK, H. 2006 Large-deformation analysis of the elastic recoil of fibre layers in a Brinkman medium with application to the endothelial glycocalyx. J. Fluid Mech. 554, 217-235.

HAPpel, J. \& Brenner, H. 1973 In Low Reynolds Number Hydrodynamics, 2nd edn, p. 393. Martinus Nijhoff. 
Hasimoto, H. 1958 On the flow of a viscous fluid past a thin screen at small Reynolds numbers. $J$. Phys. Soc. Japan 13, 633-639.

Henry, C. B. S. \& Duling, B. R. 1999 Permeation of the luminal capillary glycocalyx is determined by hyaluronan. Am. J. Physiol. 277, H508-H514.

Henry, C. B. S. \& Duling, B. R. $2000 \mathrm{TNF}-\alpha$ increases entry of macromolecules into luminal endothelial cell glycocalyx. Am. J. Physiol. 279, H2815-H2823.

Hu, X. \& Weinbaum, S. 1999 A new view of Starling's hypothesis at the microstructural level. Microvasc. Res. 58, 281-304.

Hu, X., Adamson, R. H., Liu, B., Curry, F. E. \& Weinbaum, S. 2000 Starling forces that oppose filtration after tissue oncotic pressure is increased. Am. J. Physiol. Heart Circ. Physiol. 279, H1724-H1736.

Intaglietta, M. \& De Plomb, E. P. 1973 Fluid exchange in tunnel and tube capillaries. Microvasc. Res. 6, 153-168.

Karniadakis, G. E. \& Sherwin, S. 2005 Spectral/hp Element Methods for Computational Fluid Dynamics, 2nd edn. Oxford University Press.

Levick, J. R. 1987 Flow through interstitium and other fibrous matrices. Q. J. Exp. Physiol. 72, 409-437.

Lipowsky, H. 1995 Shear stress in the circulation. In Flow-Dependent Regulation of Vascular Function (ed. J. A. Bevan, G. Kaley \& G. M. Rubanyi), pp. 28-45 Oxford University Press.

Long, D. S., Smith, M. L., Pries, A. R., Ley, K. \& Damiano, E. R. 2004 Microviscometry reveals reduced blood viscosity and altered shear rate and shear stress profiles in microvessels after hemodilution. Proc. Natl Acad. Sci. 101, 10060-10065.

LUFT, J. H. 1966 Fine structure of capillary and endocapillary layer as revealed by ruthenium red. Fed. Proc. 25, 1773-1783.

Mason, J. C., Curry, F. E. \& Michel, C. C. 1977 The effect of proteins on the filtration coefficient of individually perfused frog mesenteric capillaries. Microvasc. Res. 13, 185-204.

Michel, C. C. 1997 Starling: the formulation of his hypothesis of microvascular fluid exchange and its significance after 100 years. Exp. Physiol. 82, 1-30.

Michel, C. C. \& Curry, F. E. 1999 Microvascular permeability. Physiol. Rev. 79, 703-761.

Pahakis, M. Y., Kosky, J. R., Dull, R. O. \& Tarbell, J. M. 2007 The role of endothelial glycocalyx components in mechanotransduction of fluid shear stress. Biochem. Biophys. Res. Commun. 355, 228-233.

Phillips, C. G., Parker, K. H. \& Wang, W. 1994 A model for flow through discontinuities in the tight junction of the endothelial intercellular cleft. Bull. Math. Biol. 56, 723-741.

Pries, A. R., Secomb, T. W. \& Gaehtgens, P. 2000 The endothelial surface layer. Pflugers Arch-Eur. J. Physiol. 440, 653-666.

Priezjev, N. V. \& Troian, S. M. 2006 Influence of periodic wall roughness on the slip behaviour at liquid/solid interfaces: molecular-scale simulations versus continuum predictions. J. Fluid Mech. 554, 25-46.

SANGANI, A. S. \& ACRIVOS, A. 1982 Slow flow past periodic arrays of cylinders with application to heat transfer. Intl J. Multiphase Flow 8, 193-206.

Schulze, C. \& FirTh, J. A. 1992 The interendothelial junction in myocardial capillaries: evidence for the existence of regularly spaced, cleft-spanning structures. J. Cell Sci. 101, 647-655.

Shapiro, L., Fannon, A. M., Kwong, P. D., Thompson, A., Lehmann, M. S., Grubel, G., Legrand, J. F., Als-Nielsen, J., Colman, D. R. \& Hendrickson, W. A. 1995 Structural basis of cell-cell adhesion by cadherins. Nature 374, 327-337.

Smith, M. L., Long, D. S., Damiano, E. R. \& Ley, K. 2003 Near-wall $\mu$-PIV reveals a hydrodynamically relevant endothelial surface layer in venules in vivo. Biophys. J. 85, 637-645.

Sparrow, E. M. \& LoEFFLer, JR A. L. 1959 Longitudinal laminar flow between cylinders arranged in regular array. AIChE J. 5, 325-330.

Squire, J. M., Chew, M., Nneji, G., Neal, C., Barry, J. \& Michel, C. 2001 Quasi-periodic substructure in the microvessel endothelial glycocalyx: a possible explanation for molecular filtering? J. Struct. Biol. 136, 239-255.

Starling, E. H. 1896 On the absorption of fluids from the convective tissue spaces. J. Physiol. 19, 312-326.

Sugihara-Seki, M. 1996 The motion of an ellipsoid in tube flow at low Reynolds numbers. J. Fluid Mech. 324, 287-308. 
Sugihara-SeKI, M. 2004 Motion of a sphere in a cylindrical tube filled with a Brinkman medium. Fluid Dyn. Res. 34, 59-76.

Sugihara-SEKI, M. 2006 Transport of spheres suspended in the fluid flowing between hexagonally arranged cylinders. J. Fluid Mech. 551, 309-321.

Thi, M. M., Tarbell, J. M., Weinbaum, S. \& Spray, D. C. 2004 The role of the glycocalyx in reorganization of the actin cytoskeleton under fluid shear stress: A 'bumper-car' model. Proc. Natl Acad. Sci. 101, 16483-16488.

Truskey, G. A., Yuan, F. \& Katz, D. F. 2004 In Transport Phenomena in Biological Systems. Pearson.

Tsay, R. \& Weinbaum, S. 1991 Viscous flow in a channel with periodic cross-bridging fibres: exact solutions and Brinkman approximation. J. Fluid Mech. 226, 125-148.

Tsay, R., Weinbaum, S. \& Pfeffer, R. 1989 A new model for capillary filtration based on recent electron microscopic studies of endothelial junctions. Chem. Engng Commun 82, 67-102.

VINK, H. \& Duling, B. R. 1996 Identification of distinct luminal domains for macromolecules, erythrocytes, and leukocytes within mammalian capillaries. Circ. Res. 79, 581-589.

VINK, H. \& Duling, B. R. 2000 Capillary endothelial surface layer selectively reduces plasma solute distribution volume. Am. J. Physiol. 278, H285-H289.

Weinbaum, S. 19981997 Whitaker distinguished lecture: Models to solve mysteries in biomechanics at the cellular level; a new view of fibre matrix layers. Ann. Biomed. Engng 26, 627-643.

Weinbaum, S., Tsay, R. \& CuRry, F. E. 1992 A three-dimensional junction-pore-matrix model for capillary permeability. Microvasc. Res. 44, 85-111.

Weinbaum, S., Zhang, X., Han, Y., Vink, H. \& Cowin, S. C. 2003 Mechanotransduction and flow across the endothelial glycocalyx. Proc. Natl Acad. Sci. 100, 7988-7995.

Weinbaum, S., Tarbell, J. M. \& Damiano, E. R. 2007 The structure and function of the endothelial glycocalyx layer. Annu. Rev. Biomed. Engng 9, 121-167.

ZENG, Y. \& Weinbaum, S. 1994 Stokes flow through periodic orifices in a channel. J. Fluid Mech. 263, 207-226.

Zhang, X., Curry, F. \& Weinbaum, S. 2006 Mechanism of osmotic flow in a periodic fibre array. Am. J. Physiol. 290, H844-H852.

Zhang, X., Adamson, R. H., Curry, F. E. \& Weinbaum, S. 2006 A 1-D model to explore the effects of tissue loading and tissue concentration gradients in the revised Starling principle. $\mathrm{Am}$. J. Physiol. 291, H2950-H2964. 